\title{
CHONDROGENESIS OF MESENCHYMAL STEM CELLS IN A NOVEL HYALURONATE-COLLAGEN-TRICALCIUM PHOSPHATE SCAFFOLDS FOR KNEE REPAIR
}

\author{
F.G. Meng§, Z.Q. Zhang§, G.X. Huang, W.S. Chen, Z.J. Zhang, A.S. He and W.M. Liao* \\ Department of Joint Surgery, First Affiliated Hospital of Sun Yat-sen University, Guangzhou, \\ Guangdong 510080, China
}

$\S$ These two authors contributed equally to this work.

\begin{abstract}
Scaffolds are expected to play a key role in the induction of chondrogenesis of mesenchymal stem cells (MSCs) for cartilage tissue regeneration. Here, we report the development of a novel tricalcium phosphate-collagenhyaluronate (TCP-COL-HA) scaffold that can function as a stem cell carrier to induce chondrogenesis and promote cartilage repair, and the investigation of chondroinductive properties of scaffolds containing varying amounts of TCP, COL and HA. TCP-COL-HA scaffolds, as well as TCPCOL scaffolds at two different TCP/COL ratios (50:50 and 25:75), were evaluated for their ability to induce cartilage regeneration from rabbit mesenchymal stem cells (rMSCs) in vitro and in vivo. Chondrogenic differentiation was evaluated by sulphated glycosaminoglycan quantification, collagen type II immunohistochemistry, and qRT-PCR. Mechanical strength was evaluated by the compression test. The results showed that the TCP-COL-HA scaffolds enhanced rMSC chondrogenesis to a greater degree than did the TCP-COL scaffolds; for the latter, the scaffold with the lower TCP/COL ratio $(25: 75)$ was superior in terms of promoting rMSC chondrogenesis. Similar results were obtained in an ectopic implantation model in nude mice. In a critical-size rabbit osteochondral defect-repair model, rMSCs seeded on TCP-COL-HA scaffolds showed greater cartilage regeneration and integration into surrounding tissue than the TCP-COL groups, in which cartilage repair was more efficient at the 25:75 than at the 50:50 ratio. These results indicate that the addition of HA and different TCP/ COL ratios can affect the chondroinductive capacity of scaffolds, and suggest that the TCP-COL-HA scaffold can serve as an effective cell carrier for cartilage regeneration.
\end{abstract}

Keywords: Cartilage engineering, mesenchymal stem cell, hyaluronic acid, collagen, biomaterial, chondrogenesis.

\author{
*Address for correspondence: \\ Dr. Weiming Liao, MD, PhD \\ Department of Orthopaedic Surgery \\ First Affiliated Hospital of Sun Yat-sen University \\ \#58 Zhongshan $2^{\text {nd }}$ Road \\ Guangzhou 510080, China
}

Telephone Number: 86-20-8775-5766

FAX Number: 86-20-8733-2150

E-mail: liaowmsysu@163.com

\section{Introduction}

Damaged articular cartilage has poor self-repair capability owing to the low metabolic rate of chondrocytes (Chen et al., 2009; Hunziker, 2002; Steadman et al., 2002). Tissue engineering is considered a potential strategy for regenerating damaged tissue (Jackson and Simon, 1999). Mesenchymal stem cells (MSCs) are an especially promising tool since they can be easily isolated from bone marrow and expanded in vitro without the loss of their capacity to differentiate into various cell types, including chondrocytes and osteoblasts (Caplan, 2005).

Scaffolds also play critical roles in tissue regeneration (Langer and Vacanti, 1993), as they provide cells with a structural basis for growth and facilitate cellular activities such as proliferation and differentiation that are required for tissue repair (Hubbell, 2003). Scaffolds consisting of components found in the cellular environment are widely used in tissue engineering because of their excellent biocompatibility. Hyaluronan (HA) and collagen (COL) are both key components of the extracellular matrix (ECM) of normal articular cartilage, which provides a favourable environment for MSC chondrogenesis (Schagemann et al., 2013). Owing to its greater availability, type I COL (COLI) has often been used in engineering tissue scaffolds (Glowacki and Mizuno, 2008; Gotterbarm et al., 2006). COLI is highly biocompatible, weakly immunogenic, and provides an excellent substrate for cell attachment, migration, proliferation and differentiation (Revell and Athanasiou, 2009). COLI has demonstrated chondroinductive activity (Kuroda et al., 2007; Zhang et al., 2012; Zheng et al., 2009). However, COL products have relatively poor physical properties, including a lack of wet tensile strength, which is important because scaffolds should be designed to serve a space-filling function in a fluid environment (Bakoš et al., 1999). Tricalcium phosphate (TCP) has good biocompatibility and biodegradability and has been shown to improve the mechanical properties of COL scaffolds (Arahira and Todo, 2014; Tanaka et al., 2005), as well as having been used in scaffolds for osteochondral regeneration (Ahn et al., 2009; Marquass et al., 2010; Seo et al., 2013; Tanaka et al., 2005). Moreover, the Food and Drug Administration have approved several COL-calcium phosphate materials, such as HealosVR (Depuy), CollagraftVR (Zimmer), OssimendVR (Collagen Matrix), and MozaikVR (Integra LifeSciences), for use as a bone-void filler along with bone-marrow extract, which confirm their clinical applicability.

HA is a linear polysaccharide that occurs naturally in articular cartilage and synovial fluid and has been shown 
to influence many processes involving the ECM, including matrix formation, cell proliferation and migration, and embryonic tissue development (Goodstone et al., 2004). HA-based scaffolds are well tolerated, safe and allow faster cell infiltration - leading to more rapid tissue formation in articular cartilage defect repair (Radice et al., 2000; Solchaga et al., 2005). Photocrosslinkable HA promotes the retention of the chondrocytic phenotype as well as cartilage matrix synthesis, and accelerates healing in an osteochondral defect model (Nettles et al., 2004), whereas a fibrin/HA composite gel efficiently promoted chondrogenic differentiation of MSCs (Park et al., 2011). Therefore, HAbased scaffolds have potential therapeutic applications in the treatment of cartilage lesions (Pavesio et al., 2003).

Exogenous chondrogenic growth factors are routinely used, but have safety issues over the long term (Curran et al., 2006; Mueller et al., 2010; Stoop, 2008). For instance, direct injection of transforming growth factor (TGF)- $\beta$ resulted in side effects on the joints, including osteophytes formation, swelling and synovial hyperplasia (Curran et al., 2006; van Beuningen et al., 1998). Theoretically, the scaffold material itself could be used to induce chondrogenesis in MSCs; however, studies of chondro-conductive scaffolds are rare. In a previous study, we showed that a TCP-COL-HA scaffold can induce the chondrogenic differentiation of human mesenchymal stem cells (hMSCs) independent of TGF- $\beta$ in vitro (Meng et al., 2014). In the present study, we further improved the scaffold and examined its potential for inducing chondrogenic differentiation in MSCs to repair cartilage defect. As a control, we also synthesised scaffolds that were of two different TCP:COL ratios. The aim of this study is to report the further development of a novel TCPCOL-HA scaffold that can function as a stem cell carrier to induce chondrogenesis and promote cartilage repair, and to investigate the chondroinductive properties of scaffolds containing varying amounts of TCP, COL, and HA. We hypothesised that a novel TCP-COL-HA scaffold could function as a stem cell carrier to induce chondrogenesis and thereby promote cartilage repair; its effectiveness as an MSC carrier was investigated by assessing cartilage generation in vitro and in nude mouse and rabbit models.

\section{Materials and Methods}

\section{Scaffold synthesis}

Porous scaffolds were generated by freeze-drying followed by chemical cross-linking. For TCP-COL-HA scaffolds, HA $(2 \mathrm{wt} \%)$ in dry powdered form and TCP (25 wt \%) (both from Sigma, St. Louis, MO, USA) were added to a pure COLI solution (calf; Chuanger Ltd, Taijing, China) (73 wt \%). For 50:50 (TCP: $50 \mathrm{wt} \%$, COL: $50 \mathrm{wt} \%$ ) and 25:75 (TCP: 25 wt $\%$, COL: 75 wt $\%$ ) control scaffolds, only TCP was added to the COLI solution. After vigorous stirring, solutions were poured into moulds and frozen at $-60{ }^{\circ} \mathrm{C}$ for about $4 \mathrm{~h}$, followed by lyophilisation for $24 \mathrm{~h}$ in a freeze-dryer. Scaffolds were washed three times with $0.001 \mathrm{M}$ sodium bicarbonate solution and then crosslinked with $0.1 \mathrm{M}$ 1-ethyl-3-(3-dimethylaminopropyl) carbodiimide/ $\mathrm{N}$-hydroxysuccinimide solution at $4{ }^{\circ} \mathrm{C}$ for
$12 \mathrm{~h}$. After neutralisation with $0.1 \mathrm{M}$ glycine solution and washes with deionised water, lyophilisation was carried out over $24 \mathrm{~h}$ to generate the final scaffolds, which were cut into columns (height $\times$ diameter, $7.0 \times 3.5 \mathrm{~mm}$ ) and sterilised with ethylene oxide.

\section{Isolation and culture of rabbit MSCs (rMSCs)}

Animal experiments were approved by the Ethics Committee of the First Affiliated Hospital of Sun Yat-sen University (IRB: 2014C-028). rMSCs were harvested from the marrow of 8-week-old New Zealand White rabbits (Guangdong Medical Laboratory Animal Center, Guangdong, China) by density gradient centrifugation (Meng et al., 2014) and cultured in $\alpha$ Minimal Essential Medium ( $\alpha$-MEM) supplemented with $10 \%$ foetal bovine serum (FBS), $100 \mathrm{U} / \mathrm{mL}$ penicillin, and $100 \mu \mathrm{g} / \mathrm{mL}$ streptomycin (all from Gibco, Grand Island, NY, USA) at $37^{\circ} \mathrm{C}$ and $5 \% \mathrm{CO}_{2}$. Passage $3(\mathrm{P} 3)$ cells were used for experiments (Fig. 1).

\section{rMSC differentiation and phenotypic analysis}

Cells at passage $2\left(2.5 \times 10^{6}\right)$ were collected and cultured by micromass culture in chondrogenic medium consisting of high-glucose Dulbecco's Modified Eagle's Medium (DMEM) supplemented with $10 \mathrm{ng} / \mathrm{mL}$ TGF- $\beta 1$ (R\&D Systems, Minneapolis, MN, USA), $1 \times$ human insulin/ human transferrin/sodium selenite premix, $1 \mathrm{mM}$ sodium pyruvate, $50 \mathrm{ng} / \mathrm{mL}$ vitamin $\mathrm{C}, 100 \mathrm{nM}$ dexamethasone, and $40 \mu \mathrm{g} / \mathrm{mL}$ proline (all from Sigma, St. Louis, MO, USA) for $14 \mathrm{~d}$ as previously described (Johnstone et al., 1998). For histological analysis, cells were embedded in paraffin and $5-\mu \mathrm{m}$ sections were stained with alcian blue (Sigma). For osteogenic and adipogenic differentiation, cells were seeded in 6-well plates at a density of $10^{3}$ cells/ $\mathrm{cm}^{2}$ and cultured for $14 \mathrm{~d}$ in osteogenic or adipogenic differentiation medium (Meng et al., 2014). Cells were stained with alizarin red and oil red O (both from Sigma) to visualise osteocytes and adipocytes, respectively. For cell surface phenotyping, cells at passage 2 were detached and labelled with fluorophore-conjugated mouse anti-rabbit cluster of differentiation CD29, CD44, CD45, CD34 and CD14 antibodies (BD Biosciences, Franklin Lakes, NJ, USA) and analysed by flow cytometry with a FACS Calibur Coulter EPICS Elite system (BD Biosciences) to detect surface antigen expression.

\section{rMSC seeding onto scaffolds and 3-dimensional culture}

Scaffolds were soaked in $\alpha$-MEM, blotted dry, seeded with rMSCs at $2 \times 10^{7}$ cells $/ \mathrm{mL}$ in $80 \mu \mathrm{L} \alpha-\mathrm{MEM}$, and placed in a 24-well plate (one scaffold per well). After $2 \mathrm{~h}$ incubation at $37^{\circ} \mathrm{C}$ to allow rMSCs to diffuse into and adhere to the scaffolds, $1 \mathrm{~mL}$ of chondrogenic medium without TGF- $\beta 1$ was slowly added to each well. The culture medium was replaced every $2-3 \mathrm{~d}$.

\section{Cytotoxicity determination}

Cytotoxicity of the various scaffolds was assessed using Cell Counting Kit (CCK)-8 (Dojindo, Kumamoto, Japan). rMSCs were seeded on 24-well tissue culture polystyrene (TCPS) plates at a density of $2 \times 10^{4}$ cells $/ \mathrm{cm}^{2}$. Scaffolds 


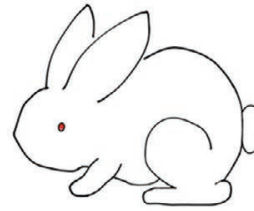

8 weeks old new zealand white rabbit
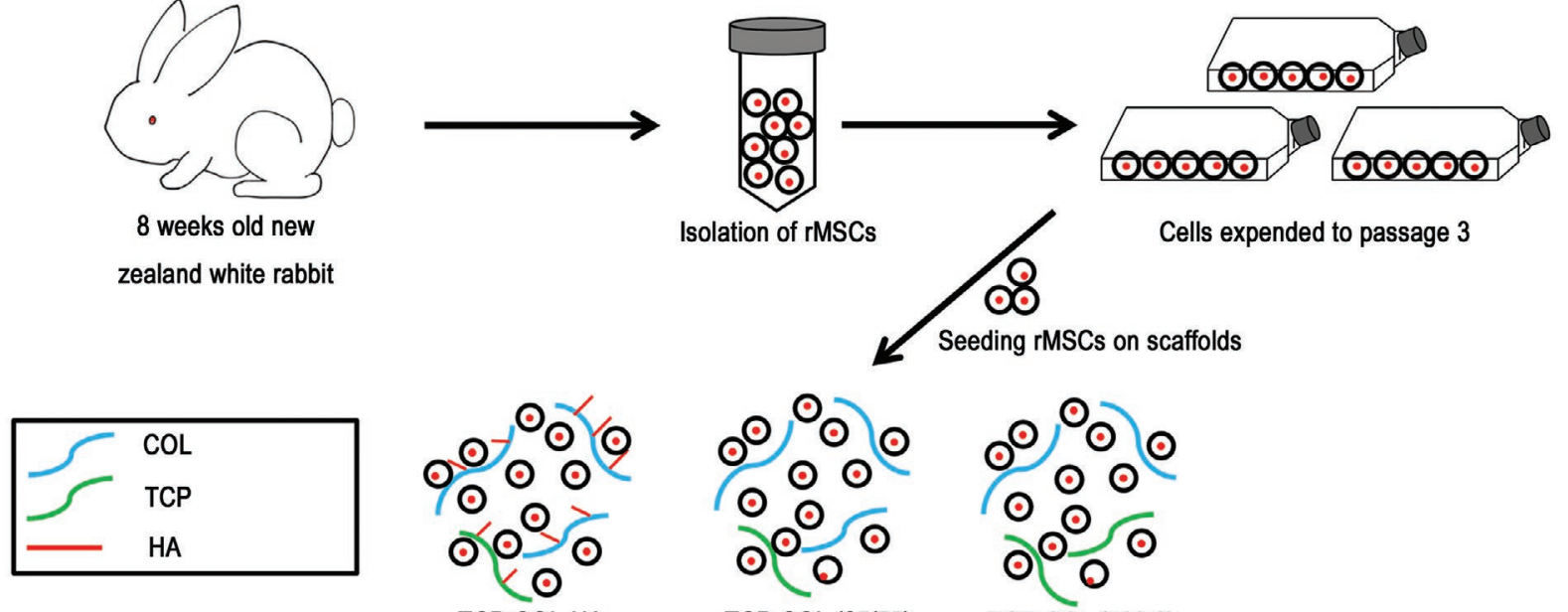

TCP-COL-HA TCP-COL (25/75)

TCP-COL $(50 / 50)$

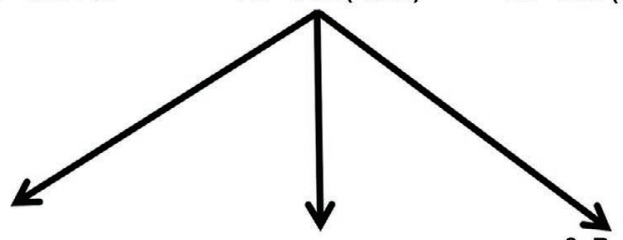

1. In vitro studies

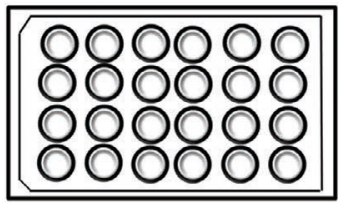

2. Subcutaneous implant

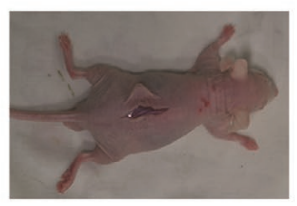

3. Rabbit osteochondro

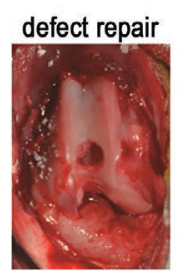

Fig. 1. Schematic illustration of the experimental protocol. rMSCs isolated from rabbit bone marrow were expanded in vitro. P3 cells were seeded on TCP-COL-HA, TCP-COL (50:50) and TCP-COL (25:75) scaffolds, which were analysed by in vitro assays and in vivo models.

were placed onto each well (one scaffold per well). rMSCs cultured in wells without scaffolds served as the control group. The rMSCs and scaffolds were cultured at $37{ }^{\circ} \mathrm{C}$ in a humidified atmosphere of $5 \% \mathrm{CO}_{2}$ for 3 and $7 \mathrm{~d}$; the original medium was then replaced with $900 \mu \mathrm{L}$ DMEM-F12 medium containing $10 \%$ FBS and $100 \mu \mathrm{L}$ CCK- 8 reagent and incubated at $37{ }^{\circ} \mathrm{C}$ for $2 \mathrm{~h}$. The supernatant containing water-soluble formazan crystals was transferred to a 96 -well plate $(100 \mu \mathrm{L} /$ well $)$, and an optical density (OD) at $450 \mathrm{~nm}$ was determined using a Tecan Sunrise microplate reader (Männedorf, Switzerland). Cell viability was normalised to that of cultures in TCPS plates as previously described (Liu et al., 2011).

\section{Scanning electron microscopy (SEM) analysis}

The morphology of rMSC-seeded scaffolds was examined by SEM. Samples incubated for $3 \mathrm{~d}$ and 3 weeks in vitro were washed twice with sterile phosphate-buffered saline (PBS, Gibco), fixed in $2.5 \%$ glutaraldehyde for $12 \mathrm{~h}$, dehydrated through a graded series of ethanol for $30 \mathrm{~min}$ at each concentration, and critical point-dried. Samples were sputter-coated with gold for 2 min before visualisation by SEM.

\section{Quantitative real-time (qRT-)PCR}

Total RNA was extracted using the Total RNA kit (Omega Bio-Tek, Norcross, GA, USA), and 500 ng were reversetranscribed using the First Strand cDNA Synthesis kit (Takara Bio, Kyoto, Japan). The cDNA served as a template for RT-PCR using SYBR Green (Toyobo Ltd., Osaka, Japan) and the primers listed in Table 1. The relative expression levels of target genes were calculated using the $2^{-\Delta \Delta \mathrm{Ct}}$ method by normalising to the level of glyceraldehyde-3-phosphate dehydrogenase. Data were normalised to the TCP-COL (50:50) group, which served as a reference.

\section{Histological analysis}

Samples were fixed with $4 \%$ paraformaldehyde (Sigma), decalcified (in the case of samples from the rabbit model), embedded in paraffin, and cut into 5 - $\mu \mathrm{m}$ sections that were deparaffinised, rehydrated, and stained with haematoxylin and eosin (H\&E) for morphological evaluation and staining with alcian blue and safranin O (both from Sigma) (in the case of samples from the rabbit model) to visualise sulphated glycosaminoglycan (GAG) distribution.

Collagen type II expression was analysed by immunohistochemistry. Sections prepared as described 
Table 1. Primers for qRT-PCR.

\begin{tabular}{|l|l|l|}
\hline Genes & \multicolumn{2}{|c|}{ Primer sequence $\left(\mathbf{5}^{\prime} \rightarrow \mathbf{3}\right.$ ') } \\
\hline COLII & Forward & GGCTTTCCTGGAGAGAAAGG \\
\hline & Reverse & ATAGAACCAGCAGGGCCAGG \\
\hline Aggrecan & Forward & AGGTCGTGGTGAAAGGTGTTG \\
\hline & Reverse & GTAGGTTCTCACGCCAGGGA \\
\hline COLI & Forward & GGCTTTCCTGGAGAGAAAGG \\
\hline & Reverse & ATAGAACCAGCAGGGCCAGG \\
\hline COLX & Forward & GAAAACCAGGCTATGGAACC \\
\hline & Reverse & GCTCCTGTAAGTCCCTGTTGC \\
\hline GAPDH & Forward & TCACCATCTTCCAGGAGCGA \\
\hline & Reverse & CACAATGCCGAAGTGGTCGT \\
\hline SOX9 & Forward & GGTGCTCAAGGGCTACGACT \\
\hline & Reverse & GGGTGGTCTTTCTTGTGCTG \\
\hline
\end{tabular}

above were pre-treated with pepsin solution for $15 \mathrm{~min}$ at $37{ }^{\circ} \mathrm{C}$ and processed with the Envision Detection kit (GeneTech, Shanghai, China) according to the manufacturer's instructions. Briefly, sections were incubated for $10 \mathrm{~min}$ with $50 \mu \mathrm{L} 3 \% \mathrm{H}_{2} \mathrm{O}_{2}$ solution, followed by incubation with mouse anti-rabbit antibody (Abcam, Cambridge, MA, USA) overnight at $4{ }^{\circ} \mathrm{C}$. Negative controls were prepared by substituting PBS for the primary antibody. A biotinylated secondary antibody that was included in the kit was applied for $30 \mathrm{~min}$. Sections were stained with 3,3'-diaminobenzidine tetrahydrochloride (supplied with the kit), counterstained with haematoxylin, dehydrated, and mounted. Immunoreactivity was analysed by determining the integrated optical density (IOD) per stained area in $\mu \mathrm{m}^{2}$ using Image-Pro Plus 6.0 software (Media Cybernetics, Rockville, MD, USA). Safranin Oand alcian blue-stained areas were also measured using the software.

\section{Biochemical assay for GAG detection}

The assay was performed by measuring the reaction between GAGs and dimethylmethylene blue (DMMB) (Sigma). Samples were collected at different time points, washed with PBS, and digested with papain solution for $16 \mathrm{~h}$ at $60{ }^{\circ} \mathrm{C}$. A $15-\mu \mathrm{L}$ aliquot of supernatant was transferred to a new tube and reacted with DMMB solution as previously described (Meng et al., 2014). Absorbance was measured at $525 \mathrm{~nm}$. Scaffolds without cells were used as a blank control whose absorbance values were subtracted from those of scaffolds with rMSCs. Total GAG content in each sample was extrapolated using a standard curve obtained with shark chondroitin sulphate (Sigma). To assess the biosynthetic activity of the rMSCs, the results of GAGs quantification were normalised to the dsDNA content. In a 96-well microplate, $100 \mu \mathrm{L}$ of the extracts were combined with $100 \mu \mathrm{L}$ of $0.7 \mu \mathrm{g} / \mathrm{mL}$ Hoechst 33258 (Sigma) in water. Fluorescence was read on a GENios Pro $^{\text {TM }}$ microplate reader (Tecan, Durham, NC, USA) at excitation and emission wavelengths of 340 and $465 \mathrm{~nm}$, respectively, and was compared to that of a certified calf thymus DNA standard (Amersham Biosciences, Piscataway, NJ, USA).

\section{Analysis of mechanical properties}

Cell-seeded scaffolds were subjected to the unconfined compression test using the elf3200 Universal Testing Machine (Bose, Eden Prairie, MN, USA). Tests were performed in displacement control mode at a rate of $3 \mathrm{~mm} /$ min. After the test, compressive stress and strain were plotted as a graph based on measured sample dimensions. Peak load was obtained from the load-displacement curve and used to calculate the compressive modulus.

\section{Scaffold implantation in nude mice}

Procedures were carried out according to the guidelines of the Ethics Committee of the First Affiliated Hospital of Sun Yat-sen University. Before implantation, P3 rMSCs $\left(2 \times 10^{7}\right.$ cells $\left./ \mathrm{mL}\right)$ were seeded on scaffolds and cultured for 2 weeks in chondrogenic medium without TGF- $\beta 1$. BALB/c-nude mice (6-8 weeks old; Sun Yat-sen University Laboratory Animal Center, Guangdong, China) were anaesthetised with $1 \%$ sodium pentobarbital in water $(1 \mathrm{~mL} / 0.1 \mathrm{~kg})$. A cell-seeded scaffold was subcutaneously implanted into the back of each animal. The incision was closed with sterile sutures and animals were returned to the housing facility where they were allowed to recover and had free access to food and water. After 4 weeks, mice were sacrificed and the scaffolds were collected. Fibrous capsules and other host tissues were carefully removed using fine, straight, toothless forceps, \#15 blade in \#3 handle under a stereoscope (10× magnification). Scaffolds were rinsed with PBS, and used for biochemical, histological and immunohistochemical analyses.

\section{Rabbit osteochondral defect repair}

New Zealand White rabbits ( 12 knees from 6 rabbits for each group; 16-18 weeks old, weighing 3.0-3.4 kg; Guangdong Medical Laboratory Animal Center, Guangdong, China) were used in this study. rMSC-seeded scaffolds were prepared and cultured in chondrogenic medium without TGF- $\beta 1$ for 2 weeks prior to implantation. Rabbits were anaesthetised with $5 \%$ sodium pentobarbital $(1 \mathrm{~mL} / \mathrm{kg})$. The knee was exposed by a medial parapatellar approach and the patella was laterally dislocated. The anterior articular surface of the distal femur was exposed and a $3.3 \mathrm{~mm}$-diameter full-thickness cylindrical osteochondral defect (2-3 mm deep) was made using an electric trephine in the articular surface of the femoral patellar groove. Rabbits were divided into the following groups $(n=6$ animals each): control (defect only) or implanted with an rMSC-seeded TCP-COL (50:50), TCP-COL (25:75), or TCP-COL-HA scaffold. Scaffolds were placed in the defect site and the wound was closed by suturing the knee joint capsule and successive layers of the skin. Rabbits were allowed to recover in individual cages. Regenerated tissue/ scaffold samples were collected 8 weeks later. Constructs, consisting of regenerated tissue at the original defect sites, as well as surrounding cartilage and bone, were removed from the joints; each sample was cut into two halves, with one used for histological analysis and the other used for biochemical assays and qRT-PCR. For the latter, the regenerated tissue/scaffold sample was carefully and precisely separated from the surrounding native cartilage 
a
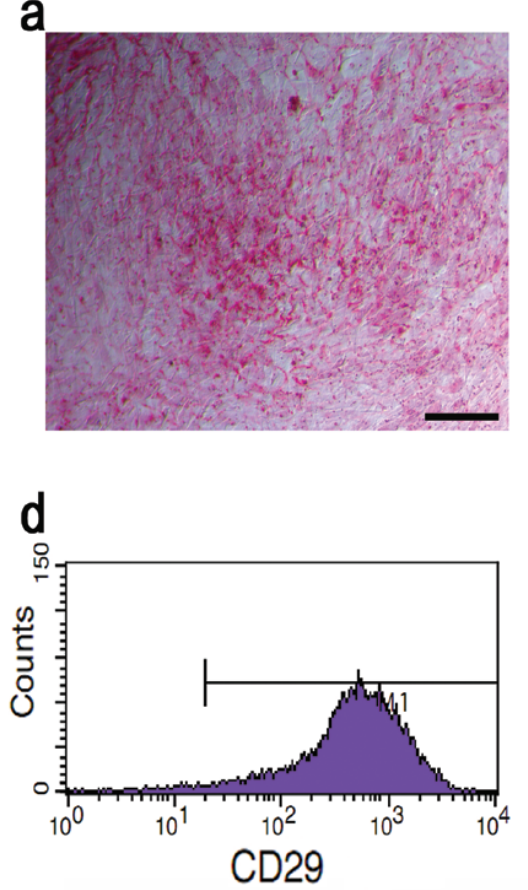

\begin{tabular}{crc} 
Marker & $\%$ Gated & Mean \\
\hline All & 100.00 & 659.40
\end{tabular}

9

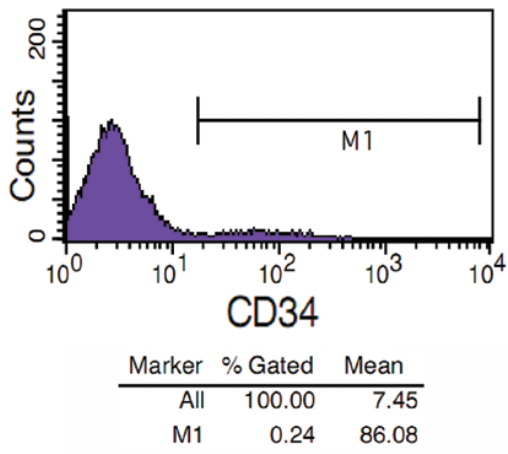

b

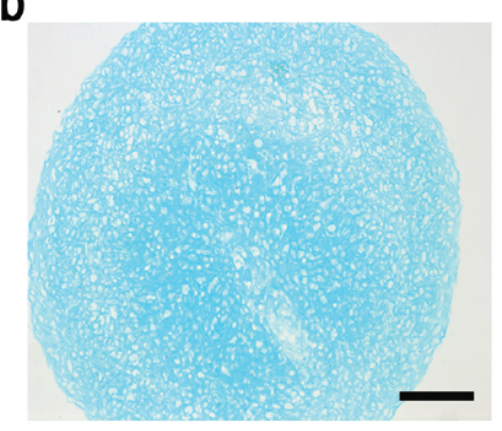

e

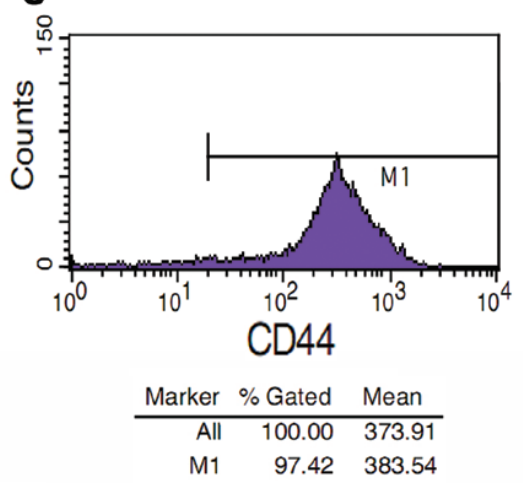

h

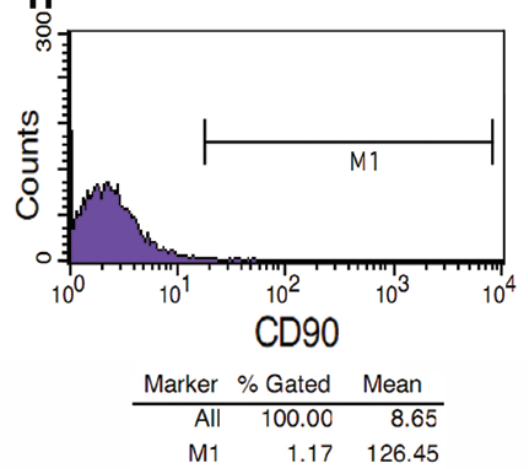

C
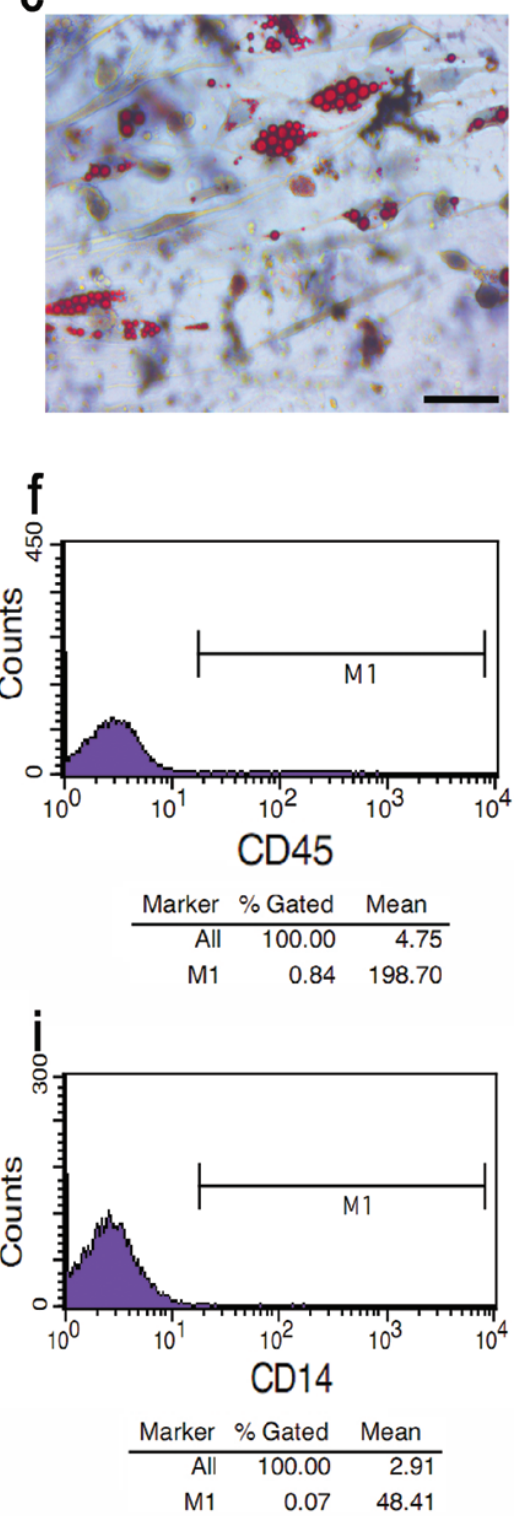

Fig. 2. Trilineage differentiation potential and phenotypic analysis of rMSCs. (a) Osteogenic, (b) chondrogenic and (c) adipogenic differentiation potential were evaluated. (d-i) rMSC cell surface antigen characterisation by flow cytometry. The MSC markers CD29 (d) and CD44 (e) were expressed, while there was no expression of the haematopoietic markers CD45 (f), CD34 (g), CD90 (h) or CD14 (i). Scale bar = $200 \mu \mathrm{m}$ (a), $50 \mu \mathrm{m}(\mathbf{b}, \mathbf{c})$.

and bone using fine, straight, toothless forceps, a \#15 surgical scalpel blade in \#3 handle under a stereoscope (10x magnification). The regenerated tissue/scaffold was rinsed with PBS and used for the biochemical assay and qRT-PCR.

\section{Statistical analysis}

Descriptive statistics were used to assess group means and determine standard deviations from at least three biological replicates. Data were evaluated by one-way analysis of variance (ANOVA) followed by Bonferroni's post hoc multiple comparisons test with $p<0.05$ considered as statistically significant. Analyses were carried out using SPSS v.13.0 for Windows (IBM Corporation, Armonk, NY, USA).

\section{Results}

\section{Trilineage differentiation potential and phenotypic} analysis

The MSC identity of cells isolated from rabbit bone marrow was confirmed by inducing their differentiation into osteogenic, chondrogenic and adipogenic lineages over $21 \mathrm{~d}$. We also verified the expression of several rabbit MSC markers by flow cytometry. Cells showed positive alizarin red, alcian blue, and oil red $\mathrm{O}$ staining (Fig. 2ac). For the phenotypic analysis, isolated cells at passage 2 were positive for the typical stem cell surface markers CD29 (98.95\%) and CD44 (97.42\%) (Fig. 2d, e), but were negative for the haematopoietic lineage markers CD45, CD34 and CD14 (Fig. 2f-i). These results confirmed that the isolated cells were MSCs. 
a

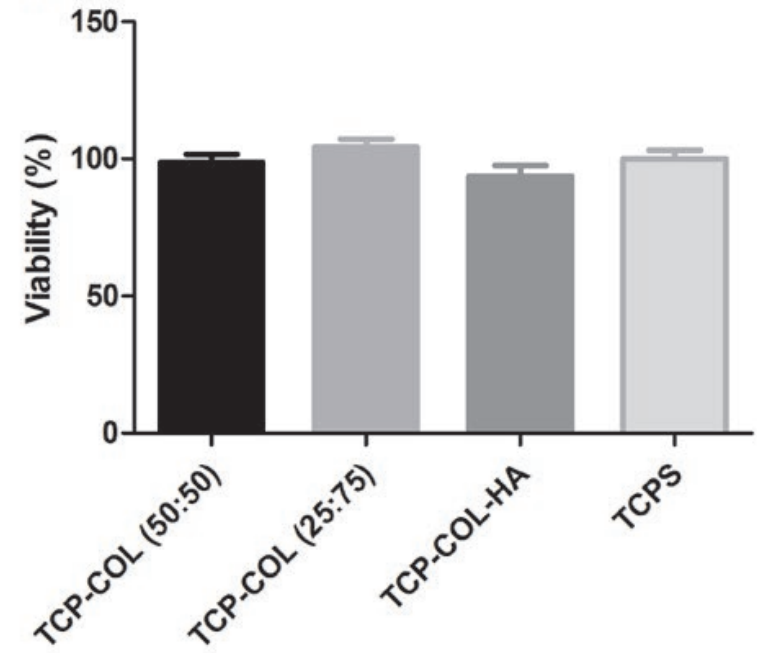

b

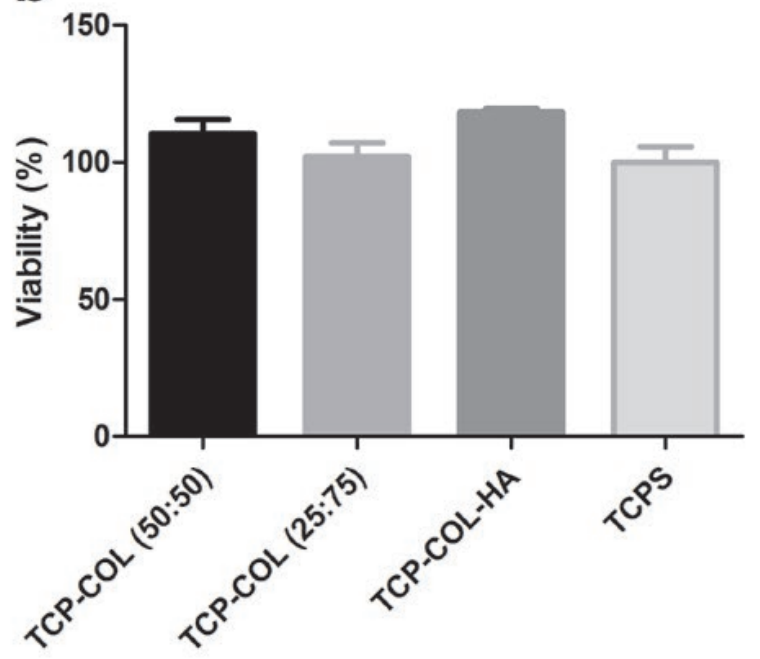

Fig. 3. Cytotoxicity of various scaffolds was assessed using CCK-8. The viability of rMSCs seeded on TCP-COL-HA, TCP-COL (50:50) and TCP-COL (25:75) scaffolds and on 24-well tissue culture polystyrene plates was evaluated at 3 and $7 \mathrm{~d}$. Data are expressed as mean \pm SE of three experiments. $* p<0.05$.

\section{Evaluation of scaffold cytotoxicity}

There was no difference in cell viability between the control, TCP-COL (50:50), TCP-COL (25:75), and TCPCOL-HA groups at 3 and $7 \mathrm{~d}$ (Fig. 3), indicating that the scaffolds were not toxic to rMSCs.

\section{Characterisation of rMSC-seeded scaffolds}

In terms of gross morphology, the TCP-COL-HA scaffold without cells has a rough and porous appearance (Fig. 3a), while the rMSC-seeded TCP-COL-HA scaffold had a smoother and glistening appearance after 3 weeks culture in vitro (Fig. $3 \mathrm{~d}$ ). There are no significant differences in appearance between TCP-COL (50:50) and rMSC-seeded TCP-COL (25:75) scaffolds (Fig. 3b, c). The appearances of both rMSC-seeded TCP-COL (50:50) and TCP-COL $(25: 75)$ are not as lustrous as that of rMSC-seeded TCPCOL-HA after 3 weeks of culture (Fig. 3b, c and d). An SEM analysis revealed that the TCP-COL-HA scaffold was highly porous, with an interconnected honeycomb-like structure (Fig. 4e, g) and pore sizes of around 50-200 $\mu \mathrm{m}$ that were suitable for rMSC culture. rMSCs were anchored and evenly distributed on the surface of the scaffold at a high density after $3 \mathrm{~d}$ of culture (Fig. 4f), and ECM secreted by rMSCs was clearly discernible after 3 weeks of culture (Fig. 4h), indicating that the scaffold had good biocompatibility.

\section{Chondrogenic gene expression analysis}

The expression of chondrogenic differentiation markers, including Aggrecan and COL II, X and I, was analysed 3 weeks after induction by qRT-PCR. At 3 weeks, cells in the TCP-COL-HA group had significantly higher expression of COL II, Aggrecan and SOX9 than those in the TCPCOL (25:75) or TCP-COL (50:50) groups, with the latter having the lowest expression (Fig. 5). In contrast, cells in the TCP-COL (50:50) group showed the highest levels of COL I and COL X at 3 weeks (Fig. 5). Taken together, these results indicate that TCP-COL-HA is more efficient at inducing rMSCs chondrogenic differentiation, whereas TCP-COL (50:50) is more efficient at inducing rMSCs osteogenic/hypertrophic differentiation in vitro.

\section{Histological, immunohistochemical and biochemical analyses}

H\&E staining was carried out to detect differences in the ECM secreted by rMSCs during differentiation. rMSCs were integrated into all three scaffolds at 3 weeks. However, ECM secretion was enhanced in cells seeded on TCP-COL-HA as compared to those grown on scaffolds lacking HA; between the latter two, cells seeded on TCPCOL $(25: 75)$ secreted more ECM than those grown on TCP-COL (50:50) at 3 weeks (Fig. 6a).

Alcian blue staining and collagen type II immunohistochemistry at 3 weeks provided evidence for chondrogenic differentiation in vitro (Fig. 6b, c). Cells seeded on TCP-COL-HA showed higher collagen type II immunoreactivity than those grown on non-HA scaffolds, with more collagen type II-positive cells observed on the TCP-COL (25:75) than on the TCP-COL (50:50) scaffold at 3 weeks. Similar results were obtained by alcian blue staining. The ratio of IOD per stained area for alcian blue staining and collagen type II immunoreactivity at 3 weeks revealed higher levels of GAG and collagen type II in the TCP-COL-HA than in the TCP-COL $(25: 75)$ or TCPCOL-HA (50:50) groups, with higher levels observed in the TCP-COL (25:75) than in the TCP-COL (50:50) group (Fig. 6d, e). At 3 weeks, rMSCs seeded on TCP-COL-HA exhibited increased $\mathrm{GAG}$ production normalised to dsDNA (GAG/dsDNA) than cells grown on TCP-COL (25:75) or TCP-COL (50:50), with the former producing more GAG/dsDNA than the latter (Fig. 6f). These observations are consistent with results of the qRT-PCR analysis, 

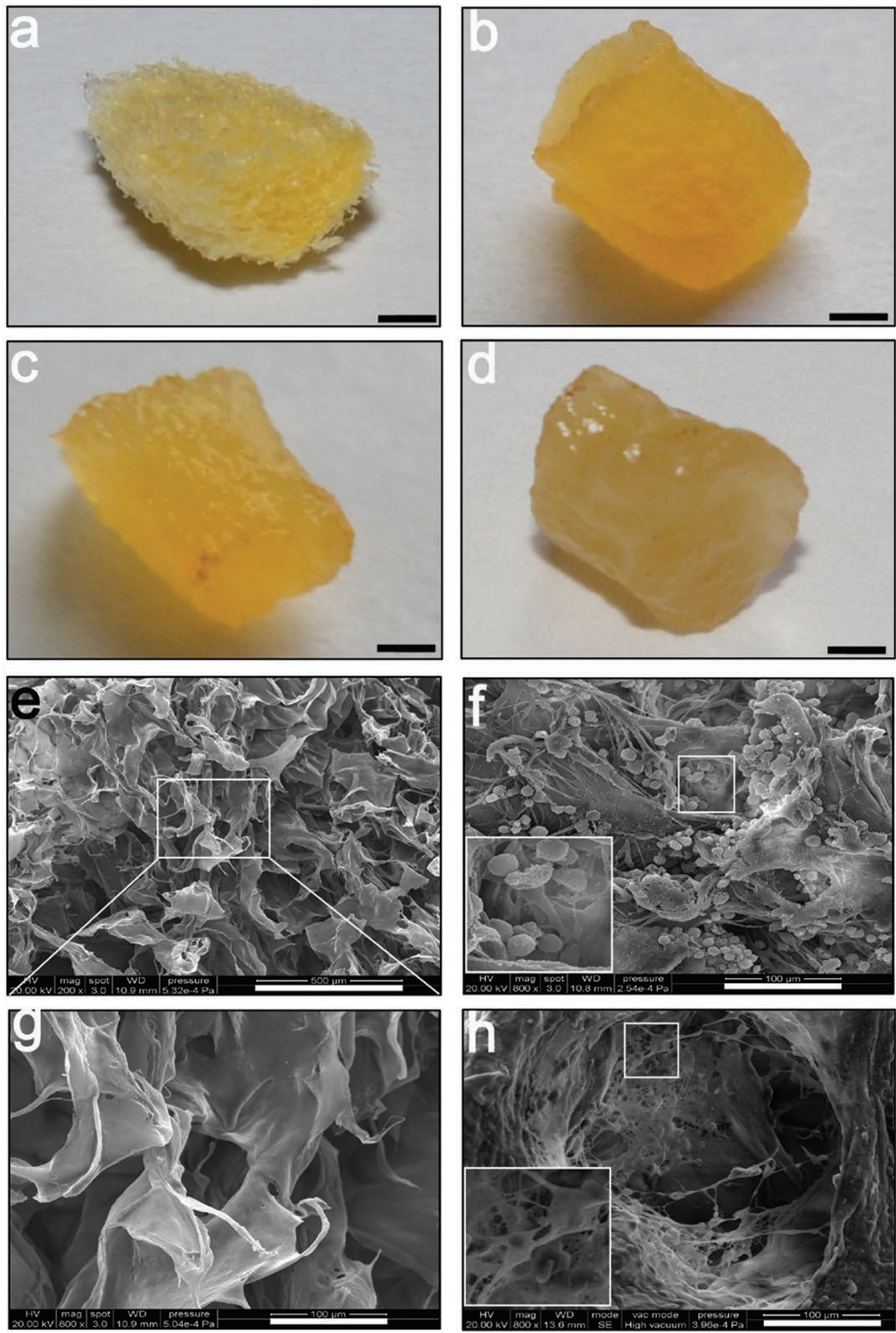

Fig. 4. Gross examination and SEM analysis of rMSC-seeded scaffolds. (a, d) Images of TCP-COL-HA scaffold without cells (a) or seeded with rMSCs after 3 weeks culture (d). (b, c, e, f) Representative micrographs of the TCPCOL-HA scaffold without cells $(\mathbf{b}, \mathbf{c})$ or seeded with rMSCs after $3 \mathrm{~d}(\mathbf{e})$ and 3 weeks $(\mathbf{f})$ of culture. Images were acquired by SEM $(200 \times$ and $800 \times)$. Scale bar $=2 \mathrm{~mm}(\mathbf{a}, \mathbf{b}, \mathbf{c}, \mathbf{d}), 500 \mu \mathrm{m}(\mathbf{e}), 100 \mu \mathrm{m}(\mathbf{f}, \mathbf{g}, \mathbf{h})$. 
a

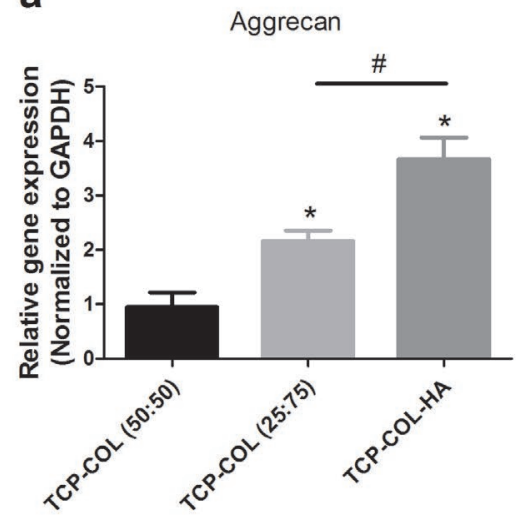

d

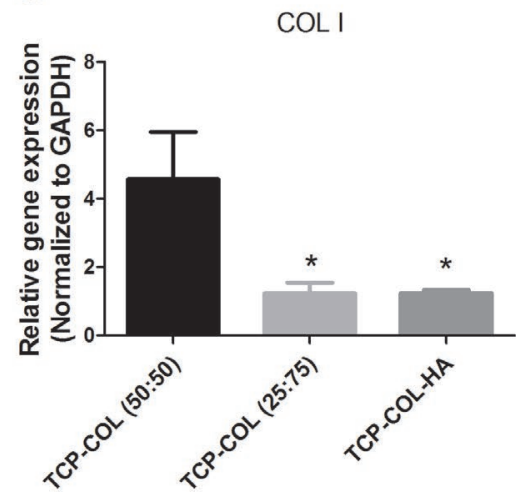

b

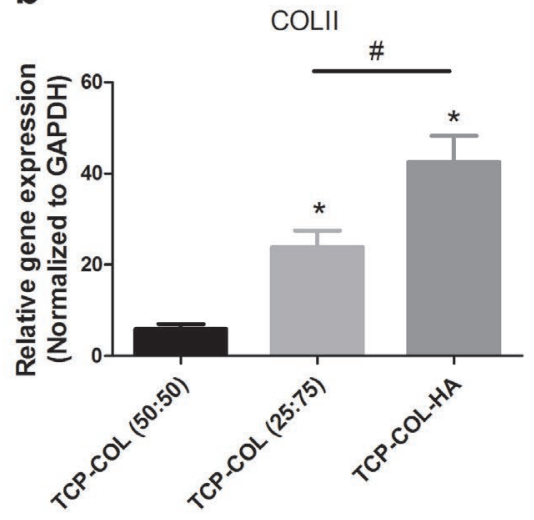

e

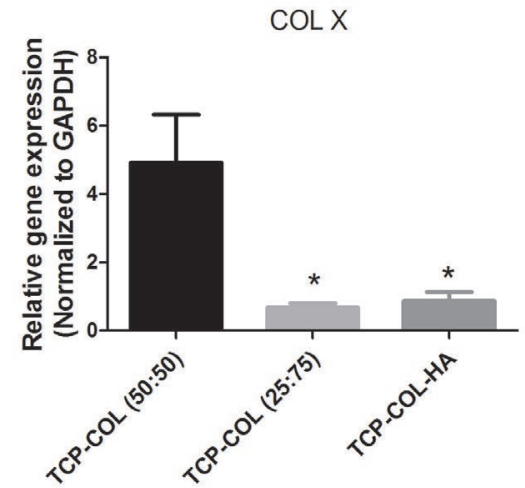

C

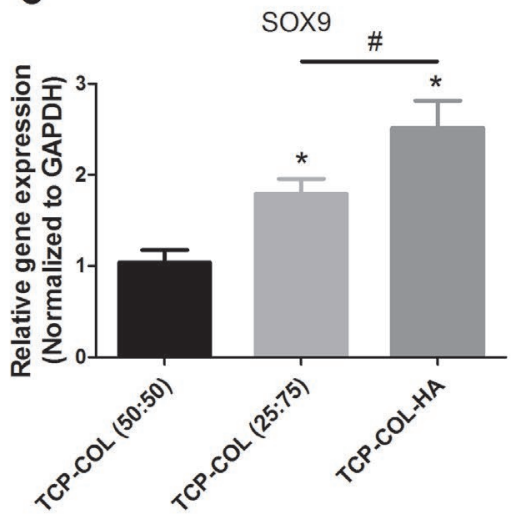

Fig. 5. qRT-PCR analyses of rMSC-seeded scaffolds. Transcript expression of aggrecan (a), COL II (b), SOX9 (c), COL I (d) and COL X (e) in rMSC-seeded TCP-COL-HA, TCP-COL (25:75) and TCP-COL (50:50) scaffolds, as determined by qRT-PCR after 1, 2 and 3 weeks of culture. Gene expression levels were normalised to that of glyceraldehyde 3-phosphate dehydrogenase (GAPDH). Data represent mean $\pm \mathrm{SE}$ of three experiments. Within a given group, significant differences $(p<0.05) v s$. TCP-COL $(50: 50)$ are denoted by $(*)$ and those between TCP-COL-HA and TCP-COL (25:75) are denoted by (\#).

indicating that TCP-COL-HA is more efficient at inducing chondrogenic differentiation of rMSCs in vitro.

\section{Analysis of mechanical properties}

Mechanical properties of rMSCs were analysed after 3 weeks of culture. The compressive modulus was higher in the TCP-COL-HA than in the TCP-COL (50:50) and TCP-COL (25:75) groups, with no significant difference observed between the latter two (Fig. 7).

\section{Gross observation and histological,} immunohistochemical, and biochemical analyses in ectopic cartilage formation assay

The regeneration efficiency of the different scaffolds was compared in an ectopic cartilage regeneration model 4 weeks after implantation. The newly formed tissueengineered cartilages showed smooth and glistening appearances, and there were no significant differences between the three groups (Fig. 8a, b). H\&E and alcian blue staining and collagen type II immunoreactivity were stronger in the TCP-COL-HA than in the TCP-COL groups (Fig. 8c-e), in which staining was more intense for the $(25: 75)$ than for the $(50: 50)$ group. These results indicate that TCP-COL-HA is the most effective substrate for promoting chondrogenesis and facilitates the secretion of chondrogenic ECM following in vivo subcutaneous implantation. A histomorphometric analysis confirmed that GAG and collagen type II were present at the highest levels in the TCP-COL-HA group, followed by the TCPCOL (25:75) and TCP-COL (50:50) (Fig. 8f, g) groups. Moreover, cells seeded on the TCP-COL-HA scaffold had the highest GAG/dsDNA, which was higher in cells grown on TCP-COL (25:75) than on TCP-COL (50:50) (Fig. 8h).

\section{Chondrogenic gene expression analysis in osteochondral repair}

The expression of the Aggrecan, COL II, SOX9, COL I and $X$ were examined 8 weeks after scaffold implantation. The highest levels of gene expression were observed in the TCP-COL-HA group, followed by the TCP-COL (25:50) and TCP-COL (50:50) groups (Fig. 9a-c). On the other hand, the TCP-COL (50:50) group showed higher expression of $C O L I$ and $X$ (Fig. 9d, e).

\section{Gross morphological and histological analyses of osteochondral repair in vivo}

In untreated control animals, the osteochondral defect was nearly devoid of tissue after 8 weeks, with only amorphous soft fibres observed in the central area (Fig. 10a) and a chondral phase that was completely denuded 


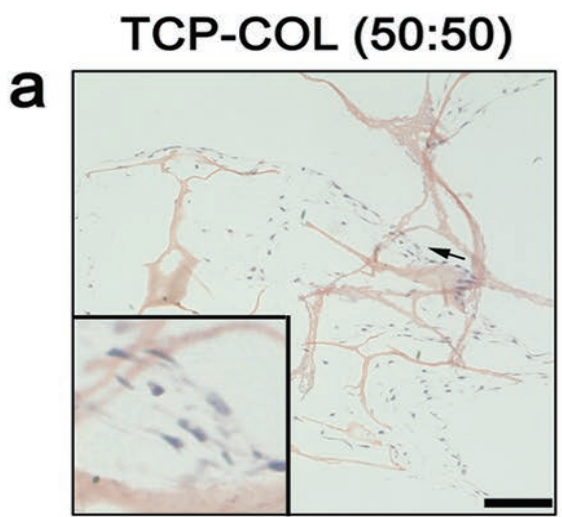

b

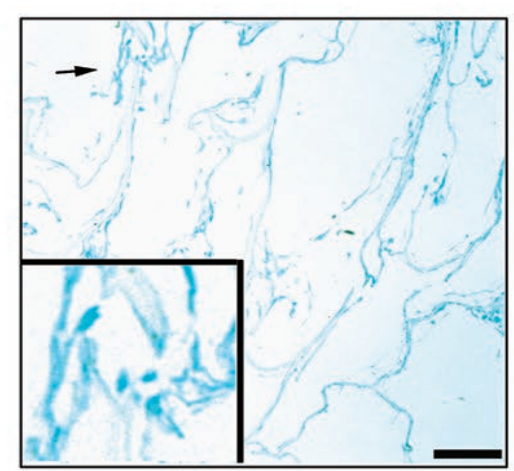

C
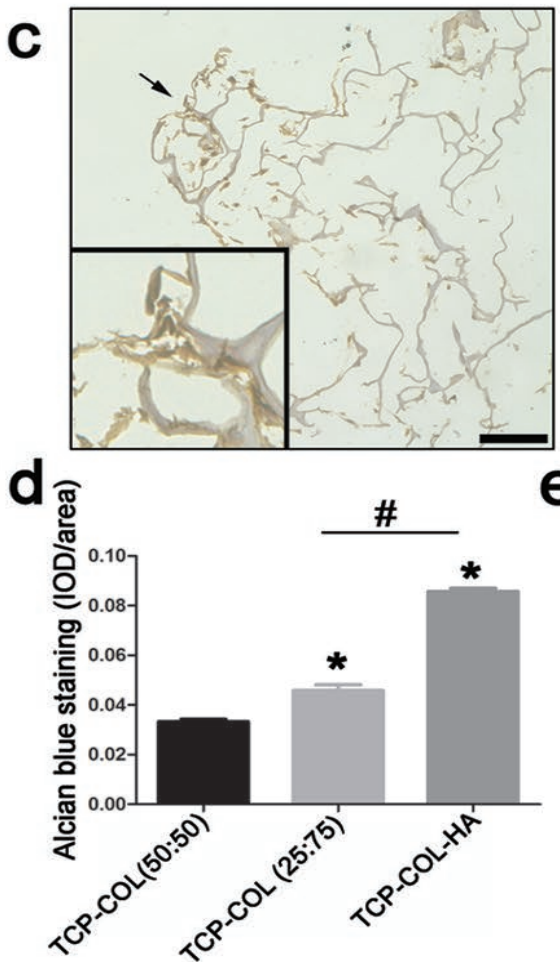

TCP-COL (25:75)
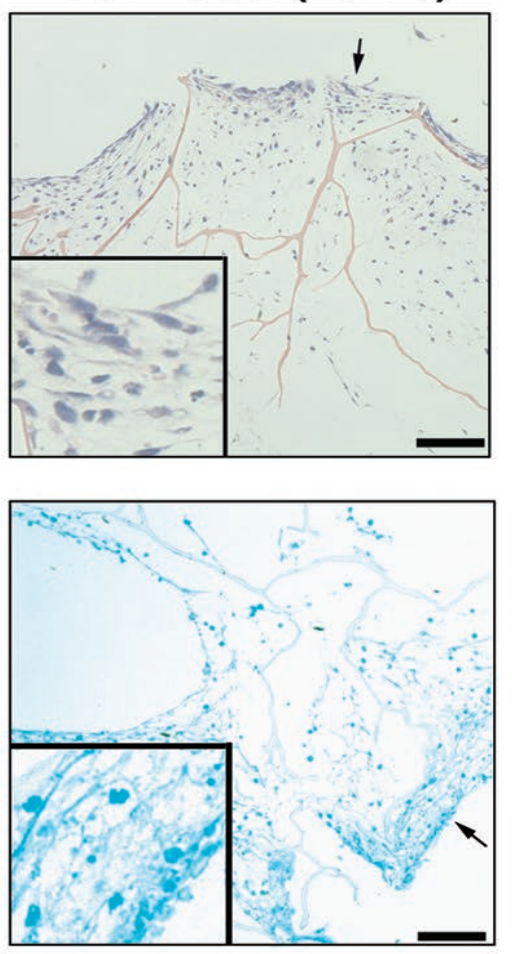

TCP-COL-HA
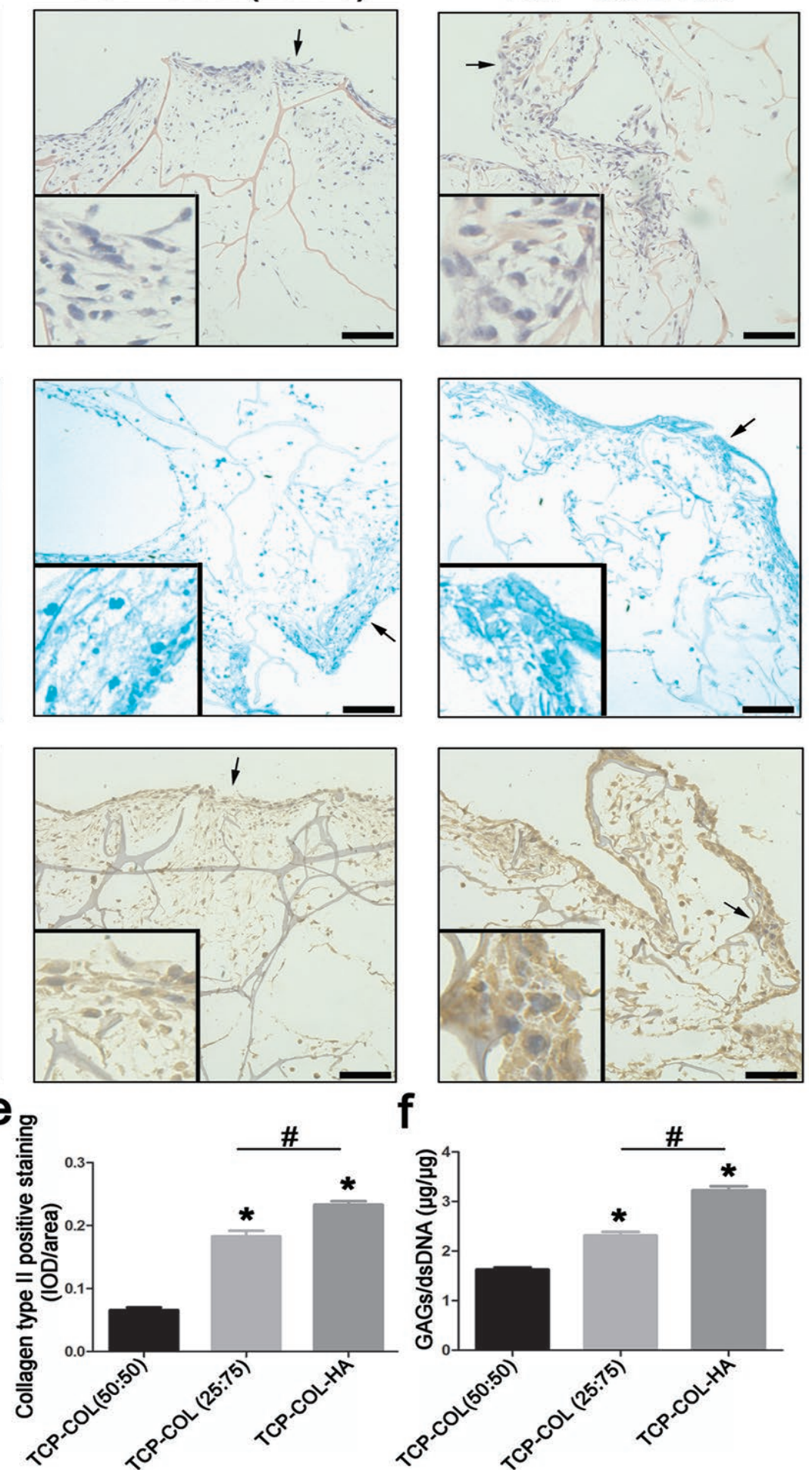
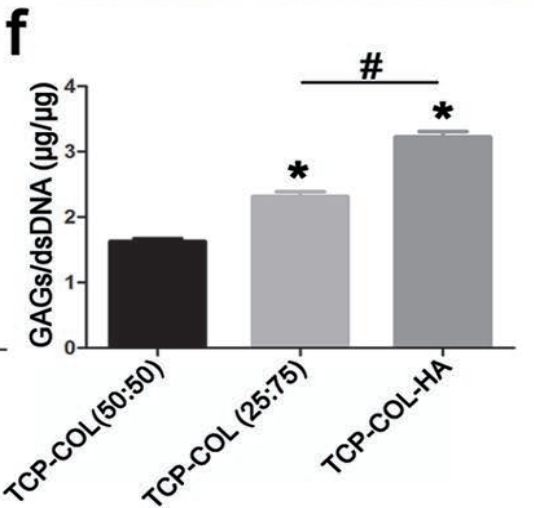

Fig. 6. Histomorphometric and biochemical analyses of rMSC-seeded scaffolds. (a-c) Histological characteristics were examined by H\&E staining (a), alcian blue staining (b) and collagen type II immunohistochemistry (c). (d, e) Average IOD/area for alcian blue staining (d) and collagen type II immunohistochemistry (e) of rMSC-seeded scaffolds after 3 weeks of culture. (f) Quantification of GAGs/dsDNA in rMSCs seeded on scaffolds at 1, 2 and 3 weeks. Data represent mean $\pm \mathrm{SE}$ of three experiments. Within a given group, significant differences $(p<0.05) v s$. TCP-COL (50:50) are denoted by (*), and those between TCP-COL-HA and TCP-COL (25:75) are denoted by (\#). Scale bar $=100 \mu \mathrm{m}$. IOD/area, integrated optical density per stained area. 


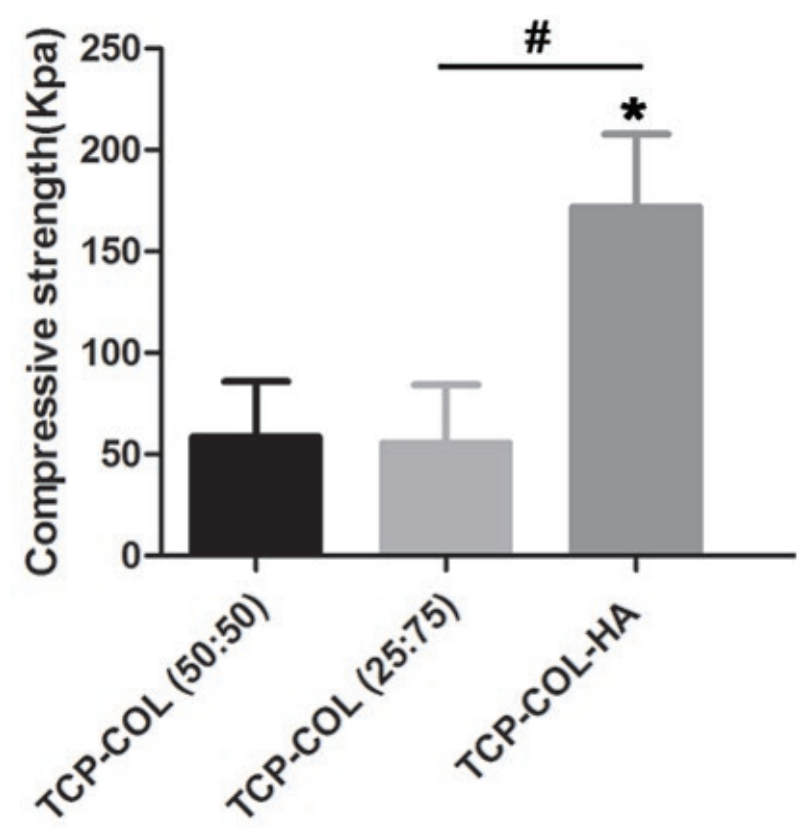

Fig. 7. Analysis of mechanical properties of rMSCseeded scaffolds. Mechanical properties were evaluated 4 weeks after seeding with rMSCs. Data represent mean $\pm \mathrm{SE}$ of three experiments. ${ }^{*} p<0.05$.

(Fig. 10e, i, m). H\&E, alcian blue and safranin O staining revealed only small amounts of newly formed cartilage in the chondral region, suggesting limited intrinsic repair capability. Marginal tissue repair was detected in the TCPCOL (50:50) group; most of the regenerated tissue within the defect was thin and rough, and some scaffold remained within the centre, although it had mostly integrated into adjacent host cartilage (Fig. 10b). The defect was filled with loose regenerated tissue and the cell-seeded scaffold. Only small areas of the defect were positive for alcian blue and safranin O staining (Fig. 10f, j, n). In the TCP-COL (25:75) group, the regenerated tissue was thicker and its surface topography was more similar to that of host cartilage, although the junction between the two was still identifiable and alcian blue and safranin $\mathrm{O}$ staining intensity was weaker than in native cartilage (Fig. 10c, g, k, o). Numerous chondrocyte-like cells surrounded by dense ECM were observed within the defect (Fig. 10s). Meanwhile, in the TCP-COL-HA group, the regenerated tissue completely occupied the defect and had a glistening white appearance, with only a pinhole remaining in the central area (Fig. 10d). The regenerated tissue was similar in colour and texture to native cartilage, as evidence by strong alcian blue and safranin O staining for GAG (Fig. 10h, 1, p). At higher magnification, the integration of the regenerated tissue into native cartilage was greater in TCP-COL-HA and TCP-COL (25:75) than in the other two groups (Fig. 10q- $t$ ), while the TCP-COL (50:50) was superior to the untreated control group. A histomorphometric analysis revealed that GAG levels were highest in the TCP-COL-HA group, followed by those in the TCP-COL $(25: 75)$ and TCP-COL (50:50) groups and untreated controls (Fig. 10u, v).
Biochemical assay for GAGs in osteochondral repair The GAG/dsDNA ratio is indicative of the quality of regenerated cartilage. The ratio was highest in the TCPCOL-HA group, followed by the TCP-COL (25:75) and TCP-COL (50:50) groups (Fig. 10w).

\section{Discussion}

In this study, we found that the TCP-COL-HA scaffold can function as a stem cell carrier to induce chondrogenesis of rMSCs in vitro and in vivo and thereby promote cartilage repair in rabbits. The TCP-COL-HA scaffold showed more cartilaginous characteristics than the TCP-COL scaffold. Of the latter, TCP-COL (25:75) was superior to TCP-COL (50:50) in terms of inducing chondrogenic differentiation of rMSCs and repairing cartilage defect.

Application of chondrogenic growth factors, especially TGF- $\beta$, is a routine strategy for inducing chondrogenesis of hMSCs, but they have safety problems in the long term. Scaffolding material has always been expected to play a crucial role in inducting chondrogenic differentiation of MSCs to generate cartilage tissue. We previously demonstrated that the TCP-COL-HA scaffold can induce chondrogenic differentiation of both ATDC5 and hMSCs without TGF- $\beta$ in vitro (Meng et al., 2014). In this study, we found that the TCP-COL-HA scaffold can induce chondrogenesis of rMSCs in vitro and in vivo and have good potential for cartilage repair. Similar results have been reported in previous studies using other materials. A natural scaffold composed of ECM produced by chondrocytes was successfully used to promote chondrogenic differentiation of rMSCs without TGF- $\beta$ treatment (Choi et al., 2010). Gelatin-chitosan hybrid materials have been reported to increase ECM synthesis and promote MSC chondrogenesis (Breyner et al., 2010). However, conflicting results were reported by other investigators using TGF- $\beta 3$ and HA in a photopolymerised hydrogel; in this case, TGF- $\beta 3$ was demonstrated to be essential for proteoglycan production (Sharma et al., 2007). The discrepancy in the results may be attributable to the HA and materials, which were different from those used in the present study. For instance, the previous study used an HA with a molecular weight of $1.1 \times 10^{6} \mathrm{Da}$, while the one used here was $2 \times 10^{6} \mathrm{Da}$. Previous studies have found that HA molecules of 1.65$3 \times 10^{6} \mathrm{Da}$ can induce chondrogenic differentiation of rMSCs (Hegewald et al., 2004). Overall, these results suggest that the growth factors may not be critical if optimal conditions for chondrogenic differentiation of MSCs are provided by scaffolds. Inducing chondrogenic differentiation of MSCs, without the need for biological factor(s), would be beneficial in terms of avoiding potentially harmful effects and reducing production costs for therapy.

HA can promote chondrogenic differentiation of MSCs in TCP-COL-HA scaffolds. The qPCR, GAG/ dsDNA, alcian blue staining and collagen type II immunohistochemical staining results (Figs.5, 6, 8-10) indicate that the scaffold containing HA more efficiently induced chondrogenic differentiation of rMSCs in vitro 


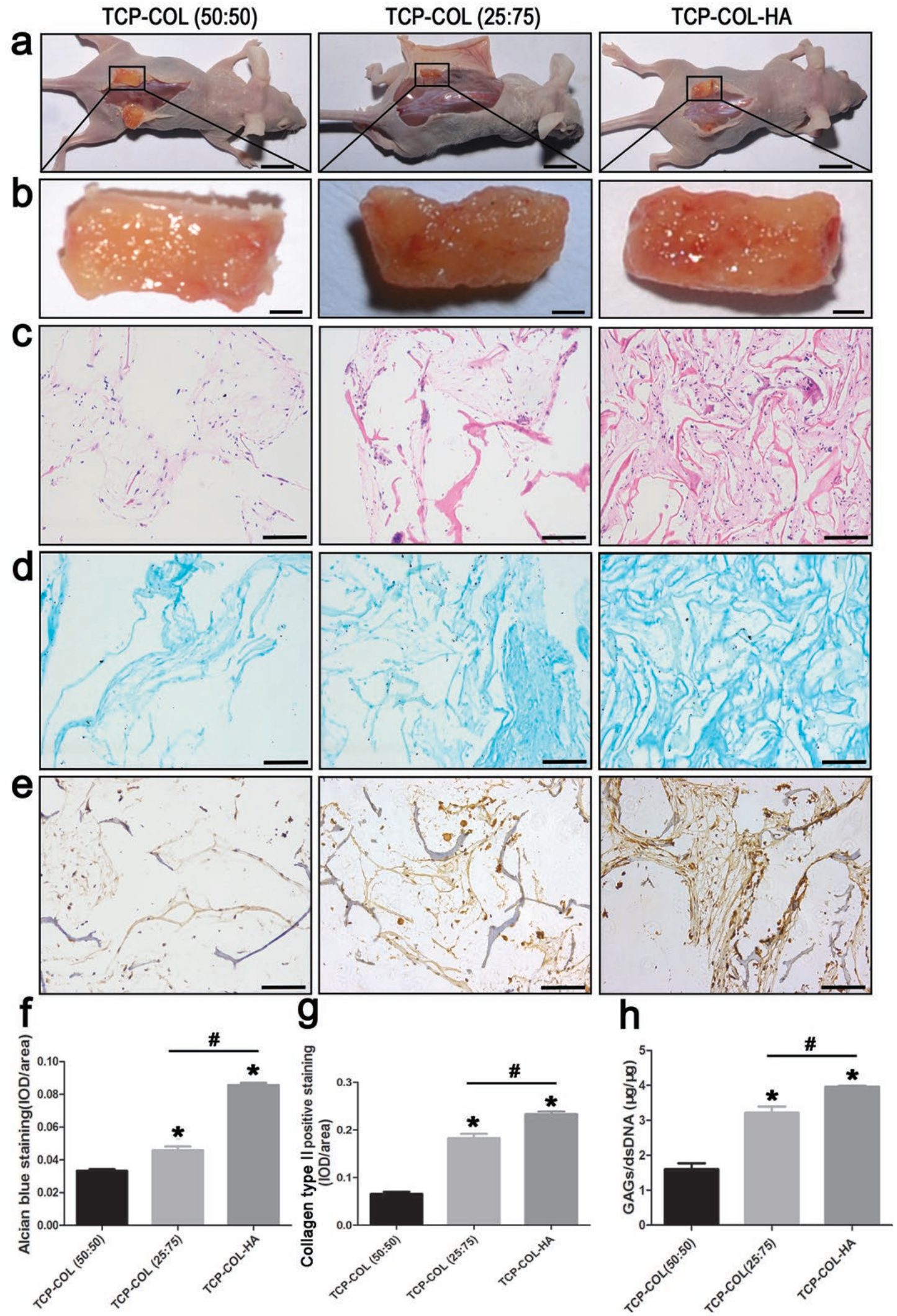

Fig. 8. Efficiency of ectopic cartilage regeneration by rMSC-seeded scaffolds in vivo. (a-e) TCP-COL-HA, TCP-COL (25:75) and TCP-COL (50:50) scaffolds seeded with rMSCs were subcutaneously implanted into the backs of nude mice, and their morphology and histological characteristics were assessed 4 weeks later by gross examination (a, b), H\&E staining (c), alcian blue staining (d) and immunohistochemical analysis of collagen type II expression (e). (f, g) Average IOD/area for alcian blue staining (f) and collagen type II immunohistochemistry (g) of rMSCs seeded on scaffolds. (h) Quantification of GAGs/dsDNA in rMSCs seeded on scaffolds 4 weeks after implantation into nude mice. Data represent mean $\pm \mathrm{SE}$ of three experiments. Within a given group, significant differences $(p<0.05) v s$. TCP-COL (50:50) are denoted by (*); those between TCP-COL-HA and TCP-COL (25:75) are denoted by (\#). Scale bar $=1 \mathrm{~cm}(\mathbf{a}), 1 \mathrm{~mm}(\mathbf{b}), 100 \mu \mathrm{m}(\mathbf{c}, \mathbf{d}, \mathbf{e})$. IOD/area, integrated optical density per stained area. 

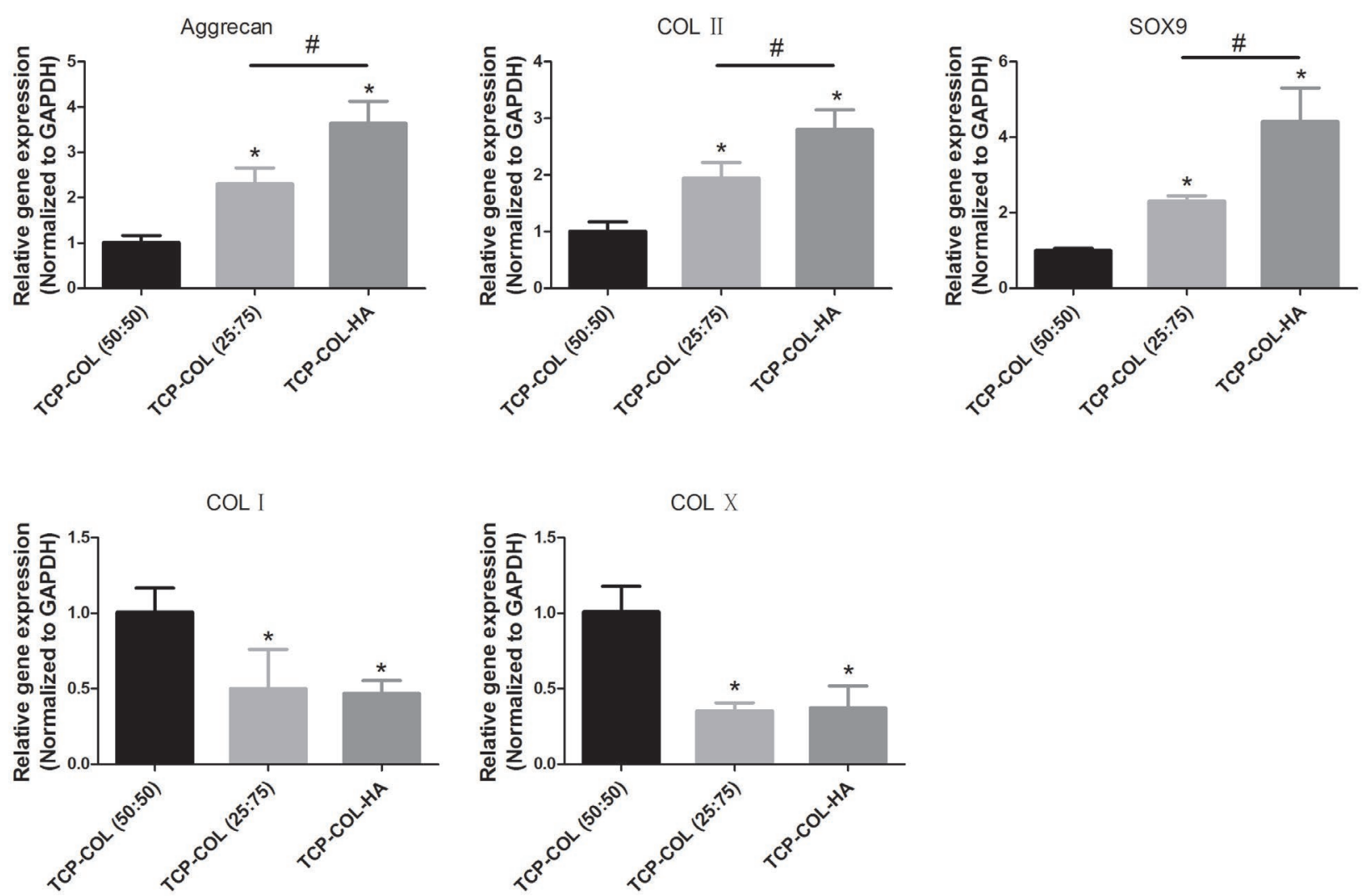

Fig. 9. Analysis of marker gene expression in implanted rMSC-seeded scaffolds. Transcript levels of (a) aggrecan, (b) COL II, (c) SOX9, (d) COL I and (e) COL X in rMSCs seeded on TCP-COL-HA, TCP-COL (25:75) and TCPCOL (50:50) scaffolds were measured by qRT-PCR 8 weeks after implantation into osteochondral defects of rabbits. Data represent mean $\pm \mathrm{SE}$ of three experiments. Within a given group, significant differences $(p<0.05) v s$. TCP-COL (50:50) are denoted by $(*)$; those between TCP-COL-HA and TCP-COL (25:75) are denoted by (\#).

and in vivo, and resulted in regenerated tissue of higher quality than those without HA, consistent with several other HA-based culture models (Park and Lee, 2014; Schagemann et al., 2013). One study found that HA added to human MSCs in alginate increased cartilage matrix production (Kavalkovich et al., 2002), suggesting a direct biological role for this molecule. The reason for enhanced chondrogenic differentiation by inclusion of HA in the scaffold is unclear, but may involve signal transduction pathways that are modulated by interactions between rMSCs and the ECM environment of TCP-COL-HA. HA interacts with the transmembrane receptor CD44 (Knudson and Loeser, 2002; Park and Lee, 2014), which mediates cell adhesion, growth and differentiation (Ishida et al., 1996; Maleski and Knudson, 1996); in the present study, before seeding on scaffolds, about $97.42 \%$ of rMSCs were positive for CD44 expression.

TCP/COL ratio may affect the chondro-inductive capacity of scaffolds. Previous studies have demonstrated that both TCP and COL scaffolds support the repair of osteochondral defects (Dawson et al., 2008; Stark et al., 2006; Tanaka et al., 2005). However, there are few studies comparing the effects of different ratios of TCP to COL in hybrid scaffolds. Our study found TCP-COL (25:75) more effectively induced chondrogenic differentiation of rMSCs in vitro and in vivo as compared to TCP-COL (50:50). In contrast, TCP-COL (50:50) more effectively induced hypertrophy during the chondrogenic differentiation of rMSCs. Two mechanisms are proposed to account for this observation. Firstly, some studies have shown that type I COL hydrogels can induce chondrogenesis in vitro (Zhang et al., 2012) and in vivo (Kuroda et al., 2007; Zheng et al., 2009). Secondly, TCP-containing materials have the potential to increase calcium concentration outside of MSCs, which is critical for mineralisation of osteoblast-like cells (Chang et al., 2000; Matsuoka et al., 1999), which likely contributes to hypertrophy during chondrogenic differentiation of rMSCs.

The mechanical integrity of a scaffold plays an important role in its effectiveness as a biomaterial. COL products have inadequate mechanical properties, which are important for tissues that are continuously subjected to compressive forces in vivo (Bakoš et al., 1999). The addition of TCP enhances the mechanical properties of COL scaffolds (Arahira and Todo, 2014); as such, it is important to compare materials with different $\mathrm{TCP} / \mathrm{COL}$ ratios to determine the ideal balance between chondrogenesisinducing capability and good mechanical properties. In our study, the TCP-COL (25:75) scaffold promoted cartilage formation to a greater degree than the TCP-COL (50:50) scaffold; however, there was no significant difference in the mechanical strength after 3 weeks culture in vitro between the two types of scaffold seeded with rMSCs. This may be due to increased ECM, GAGs and type II COL formation 

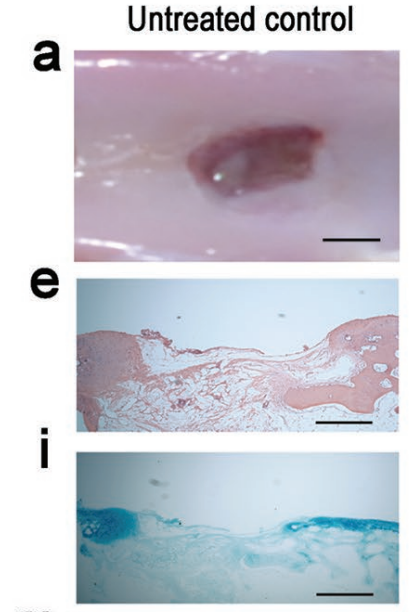

m

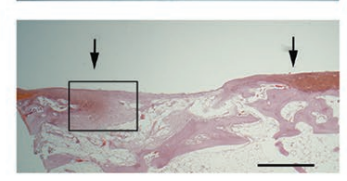

$\mathbf{q}$

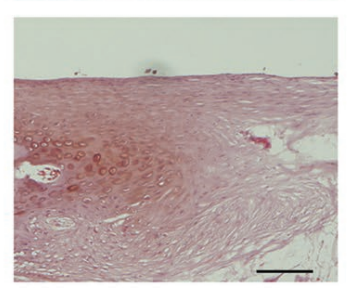

U

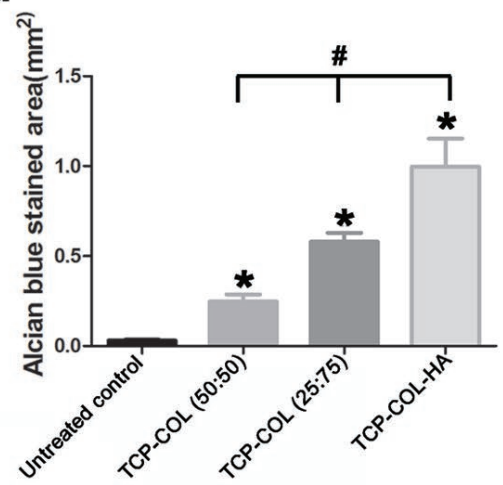

f

$\mathbf{r}$
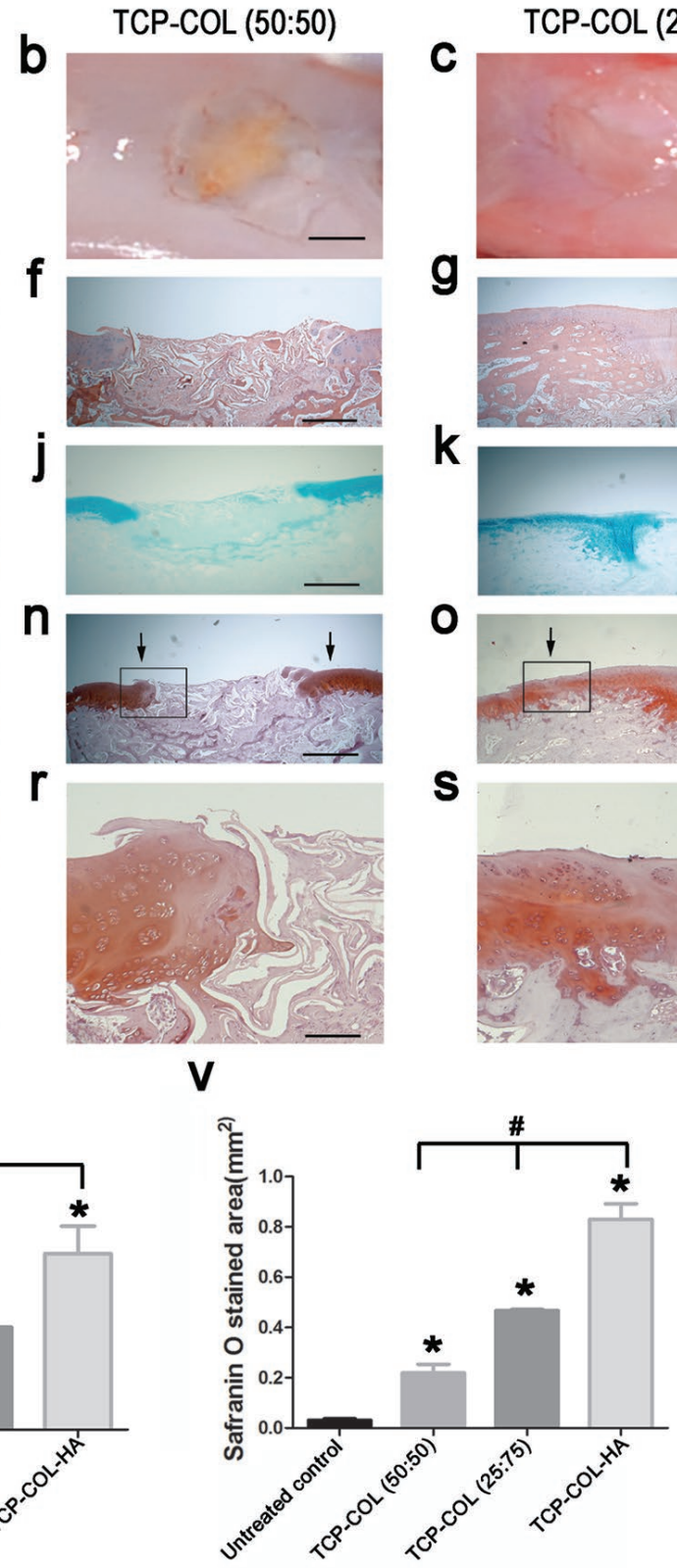

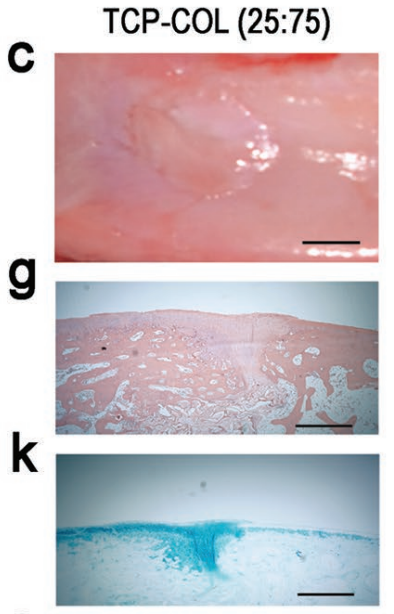

0

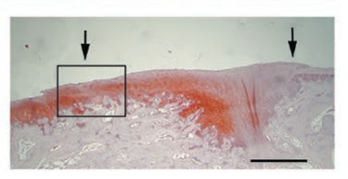

$\mathbf{S}$

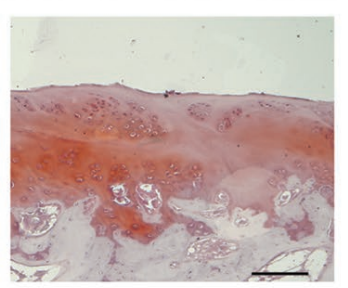

W
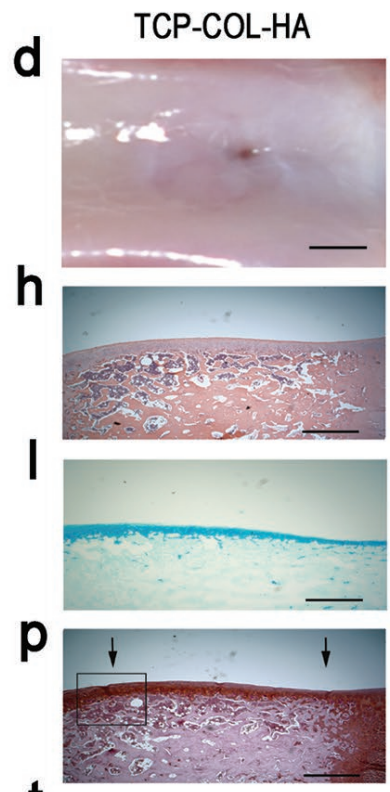

$\mathbf{t}$
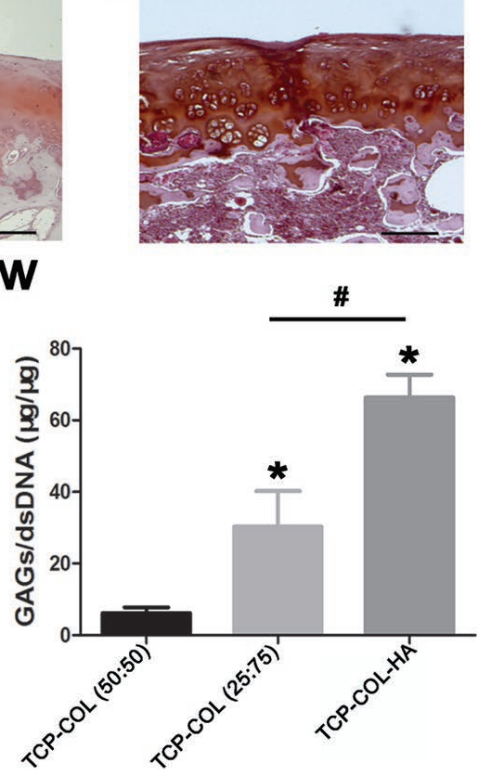

Fig. 10. Evaluation of rabbit osteochondral defect repair 8 weeks after scaffold implantation. (a-d) Gross appearance of defects in the untreated control (a), TCP-COL (50:50) (b), TCP-COL (25:75) (c) and TCP-COL-HA (d) groups. (e-p) Tissue specimens were stained with H\&E (e-h), alcian blue (i-l) and safranin O (m-p). (q-t) Magnified view of areas enclosed by black boxes in $(\mathbf{m}-\mathbf{p})$. (u, v) Histomorphometric analyses of areas stained with alcian blue (u) and safranin $\mathrm{O}(\mathbf{v})$. (w) Quantification of GAGs/dsDNA in rMSCs seeded on scaffolds 8 weeks after implantation. Data represent mean \pm standard error of three experiments. Within a given group, significant differences $(p<0.05)$ $v s$. TCP-COL (50:50) are denoted by $(*)$; those between TCP-COL-HA and TCP-COL (25:75) are denoted by (\#). Scale bar $=1 \mathrm{~mm}(\mathbf{a}-\mathbf{p}), 200 \mu \mathrm{m}(\mathbf{q}-\mathbf{t})$.

(Fig. 6), which have been shown to increase the mechanical strength of scaffolds (Tigli et al., 2011; Williamson et al., 2001; Wilson et al., 2007). The TCP-COL-HA (25:73:2) scaffold had the highest compressive modulus among all substrates tested in our study. This may be due to the presence of HA; it was previously shown that $2 \% \mathrm{HA}$ enhanced the mechanical properties of tissue-engineered cartilage constructs (Levett et al., 2014), and that addition of exogenous high molecular weight HA to cell-seeded COL gels increased tensile strength (Allison et al., 2009). Further studies are needed to clarify the exact relationship between HA in the mechanical properties of scaffolds.

The present study had limitations. We implanted rMSC-seeded scaffolds subcutaneously into nude mice and assessed chondrogenesis after four weeks only, but not eight. Although a longer subcutaneous implantation period in nude mice may be better for evaluating longterm effects, a four-week subcutaneous implantation time has been widely used in recent cartilage tissue regeneration studies (Dawson et al., 2008; Bae et al., 2010; Jung et al., 2010; Pelttari et al., 2006; Xue et al., 2012). Another limitation is that the effect of host tissue 
carryover during harvesting on qPCR and GAG/dsDNA data for subcutaneous and osteochondral samples may exist. The nude mice subcutaneous implantation model and rabbit osteochondral defect models have been widely used for various purposes in cartilage tissue engineering research (De Bari et al., 2004; Kim et al., 2011; Lam et al., 2014; Liu et al., 2011; Pelttari et al., 2006). In our study, certain prevention measures have been taken to control for the effect of host tissue carryover. For the subcutaneous and osteochondral samples, host tissues were carefully removed using fine, straight, toothless forceps, small curved, toothless forceps, \#15 blade in \#3 handle under a stereoscope (10× magnification) from the implanted samples to limit the presence of host tissues in our samples. For biochemical assays and qRT-PCR, we mainly harvested the sample from the middle of the regenerative tissue, to avoid getting marginal parts (the integration part of the regenerated tissue into native tissue) in order to prevent the presence of host tissues in our samples.

In our next study, we will use more control scaffolds (e.g., COL-HA, COL, HA, TCP, TCP-HA and TCP$\mathrm{COL}$ scaffolds in various ratios) to examine in greater detail the mechanism of the TCPCOL-HA scaffold in inducing chondrogenic differentiation of MSCs in order to further improve its effectiveness. Additionally, we are also considering using the TCP-COL-HA biomaterials in an intraoperative setup that circumvents the need for cell expansion so that the scaffold can, for example, be used in combination with marrow stimulation techniques, which are regularly used in clinics and would enhance the translational potential of the scaffold.

\section{Conclusion}

In conclusion, TCP-COL-HA induced the chondrogenesis of rMSCs and facilitated the repair of osteochondral defects. The addition of HA and different TCP/COL ratios affected the chondro-inductive effects of the scaffold. These findings suggest that the novel TCP-COL-HA scaffold might help to improve cartilage tissue engineering with safety and economic benefit, and be worthy of further investigation towards aimed clinical applications.

\section{Acknowledgments}

We thank Gang Wu (South China University of Technology, Guangzhou, China) for providing scaffold materials and technical assistance. This work was supported by the National Nature Science Foundation of China (81572119 $\&$ 81472101), and the Guangdong Provincial Natural Science Foundation of China (2014A030313064 \& 2014A030313186). We confirm that the authors have no conflicts of interest to declare and there has been no significant financial support for this work that could influence its outcome.

\section{References}

Ahn J, Lee T, Oh J, Kim S, Kim H, Park I, Choi B, Im G (2009) A novel hyaluronate-atelocollagen/ $\beta$ TCP-hydroxyapatite biphasic scaffold for the repair of osteochondral defects in rabbits. Tissue Eng Part A 15: 2595-2604.

Allison DD, Braun KR, Wight TN, Grande-Allen KJ (2009) Differential effects of exogenous and endogenous hyaluronan on contraction and strength of collagen gels. Acta Biomater 5: 1019-1026.

Arahira T, Todo M (2014) Effects of proliferation and differentiation of mesenchymal stem cells on compressive mechanical behavior of collagen/beta-TCP composite scaffold. J Mech Behav Biomed Mater 39: 218-230.

Bae SE, Choi DH, Han DK, Park K (2010) Effect of temporally controlled release of dexamethasone on in vivo chondrogenic differentiation of mesenchymal stromal cells. J Control Release 143: 23-30.

Bakoš D, Soldán M, Hernández-Fuentes I (1999) Hydroxyapatite-collagen-hyaluronic acid composite. Biomaterials 20: 191-195.

Breyner NM, Hell RC, Carvalho LR, Machado CB, Peixoto FI, Valerio P, Pereira MM, Goes AM (2010) Effect of a three-dimensional chitosan porous scaffold on the differentiation of mesenchymal stem cells into chondrocytes. Cells Tissues Organs 191: 119-128.

Caplan AI (2005) Review: mesenchymal stem cells: cell-based reconstructive therapy in orthopedics. Tissue Eng 11: 1198-1211.

Chang YL, Stanford CM, Keller JC (2000) Calcium and phosphate supplementation promotes bone cell mineralization: implications for hydroxyapatite (HA)enhanced bone formation. J Biomed Mater Res 52: 270278.

Chen H, Sun J, Hoemann CD, Lascau-Coman V, Ouyang W, McKee MD, Shive MS, Buschmann MD (2009) Drilling and microfracture lead to different bone structure and necrosis during bone-marrow stimulation for cartilage repair. J Orthop Res 27: 1432-1438.

Choi KH, Choi BH, Park SR, Kim BJ, Min BH (2010) The chondrogenic differentiation of mesenchymal stem cells on an extracellular matrix scaffold derived from porcine chondrocytes. Biomaterials 31: 5355-5365.

Curran JM, Chen R, Hunt JA (2006) The guidance of human mesenchymal stem cell differentiation in vitro by controlled modifications to the cell substrate. Biomaterials 27: 4783-4793.

Dawson JI, Wahl DA, Lanham SA, Kanczler JM, Czernuszka JT, Oreffo RO (2008) Development of specific collagen scaffolds to support the osteogenic and chondrogenic differentiation of human bone marrow stromal cells. Biomaterials 29: 3105-3116.

De Bari C, Dell'Accio F, Luyten FP (2004) Failure of in vitro-differentiated mesenchymal stem cells from the synovial membrane to form ectopic stable cartilage in vivo. Arthritis Rheum 50: 142-150.

Glowacki J, Mizuno S (2008) Collagen scaffolds for tissue engineering. Biopolymers 89: 338-344.

Goodstone NJ, Cartwright A, Ashton B (2004) Effects of high molecular weight hyaluronan on chondrocytes 
cultured within a resorbable gelatin sponge. Tissue Eng 10: $621-631$.

Gotterbarm T, Richter W, Jung M, Berardi VS, MainilVarlet P, Yamashita T, Breusch SJ (2006) An in vivo study of a growth-factor enhanced, cell free, two-layered collagen-tricalcium phosphate in deep osteochondral defects. Biomaterials 27: 3387-3395.

Hegewald AA, Ringe J, Bartel J, Krüger I, Notter M, Barnewitz D, Kaps C, Sittinger M (2004) Hyaluronic acid and autologous synovial fluid induce chondrogenic differentiation of equine mesenchymal stem cells: a preliminary study. Tissue Cell 36: 431-438.

Hubbell JA (2003) Materials as morphogenetic guides in tissue engineering. Curr Opin Biotechnol 14: 551-558.

Hunziker EB (2002) Articular cartilage repair: basic science and clinical progress. A review of the current status and prospects. Osteoarthritis Cartilage 10: 432-463.

Ishida T, Iijima T, Moriyama S, Nakamura C, Kitagawa T, Machinami R (1996) Intra-articular calcifying synovial sarcoma mimicking synovial chondromatosis. Skeletal Radiol 25: 766-769.

Jackson DW, Simon TM (1999) Tissue engineering principles in orthopaedic surgery. Clin Orthop Relat Res 367 Suppl: S31-45.

Johnstone B, Hering TM, Caplan AI, Goldberg VM, Yoo JU (1998) In vitro chondrogenesis of bone marrowderived mesenchymal progenitor cells. Exp Cell Res 238: 265-272.

Jung HH, Park K, Han DK (2010) Preparation of TGFbeta1-conjugated biodegradable pluronic F127 hydrogel and its application with adipose-derived stem cells. J Control Release 147: 84-91.

Kavalkovich KW, Boynton RE, Murphy JM, Barry F (2002) Chondrogenic differentiation of human mesenchymal stem cells within an alginate layer culture system. In Vitro Cell Dev Biol Anim 38: 457-466.

Kim M, Kim SE, Kang SS, Kim YH, Tae G (2011) The use of de-differentiated chondrocytes delivered by a heparin-based hydrogel to regenerate cartilage in partialthickness defects. Biomaterials 32: 7883-7896.

Knudson W, Loeser RF (2002) CD44 and integrin matrix receptors participate in cartilage homeostasis. Cell Mol Life Sci 59: 36-44.

Kuroda R, Ishida K, Matsumoto T, Akisue T, Fujioka H, Mizuno K, Ohgushi H, Wakitani S, Kurosaka M (2007) Treatment of a full-thickness articular cartilage defect in the femoral condyle of an athlete with autologous bonemarrow stromal cells. Osteoarthritis Cartilage 15: 226-231.

Lam J, Lu S, Lee EJ, Trachtenberg JE, Meretoja VV, Dahlin RL, van den Beucken JJ, Tabata Y, Wong ME, Jansen JA, Mikos AG, Kasper FK (2014) Osteochondral defect repair using bilayered hydrogels encapsulating both chondrogenically and osteogenically pre-differentiated mesenchymal stem cells in a rabbit model. Osteoarthritis Cartilage 22: 1291-1300

Langer R, Vacanti JP (1993) Tissue engineering. Science 260: 920-926.

Levett PA, Hutmacher DW, Malda J, Klein TJ (2014) Hyaluronic acid enhances the mechanical properties of tissue-engineered cartilage constructs. PLoS One 9: e113216.
Liu X, Jin X, Ma PX (2011) Nanofibrous hollow microspheres self-assembled from star-shaped polymers as injectable cell carriers for knee repair. Nat Mater 10: 398-406.

Maleski MP, Knudson CB (1996) Matrix accumulation and retention in embryonic cartilage and in vitro chondrogenesis. Connect Tissue Res 34: 75-86.

Marquass B, Somerson JS, Hepp P, Aigner T, Schwan S, Bader A, Josten C, Zscharnack M, Schulz RM (2010) A novel MSC-seeded triphasic construct for the repair of osteochondral defects. J Orthop Res 28: 1586-1599.

Matsuoka H, Akiyama H, Okada Y, Ito H, Shigeno C, Konishi J, Kokubo T, Nakamura T (1999) In vitro analysis of the stimulation of bone formation by highly bioactive apatite- and wollastonite-containing glass-ceramic: released calcium ions promote osteogenic differentiation in osteoblastic ROS17/2.8 cells. J Biomed Mater Res 47: 176-188.

Meng F, He A, Zhang Z, Zhang Z, Lin Z, Yang Z, Long Y, Wu G, Kang Y, Liao W (2014) Chondrogenic differentiation of ATDC5 and hMSCs could be induced by a novel scaffold-tricalcium phosphate-collagen-hyaluronan without any exogenous growth factors in vitro. J Biomed Mater Res A 102: 2725-2735.

Mueller MB, Fischer M, Zellner J, Berner A, Dienstknecht T, Prantl L, Kujat R, Nerlich M, Tuan RS, Angele P (2010) Hypertrophy in mesenchymal stem cell chondrogenesis: effect of TGF-beta isoforms and chondrogenic conditioning. Cells Tissues Organs 192: 158-166.

Nettles DL, Vail TP, Morgan MT, Grinstaff M., Setton LA (2004) Photocrosslinkable hyaluronan as a scaffold for articular cartilage repair. Ann Biomed Eng 32: 391-397.

Park H, Lee KY (2014) Cartilage regeneration using biodegradable oxidized alginate/hyaluronate hydrogels. J Biomed Mat Res 102: 4519-4525

Park SH. Choi BH, Park SR, Min BH (2011) Chondrogenesis of rabbit mesenchymal stem cells in fibrin/ hyaluronan composite scaffold in vitro. Tissue Eng Part A 17: $1277-1286$.

Pavesio A, Abatangelo G, Borrione A, Brocchetta D, Hollander AP, Kon E, Torasso F, Zanasi S, Marcacci M (2003) Hyaluronan-based scaffolds (Hyalograft C) in the treatment of knee cartilage defects: preliminary clinical findings. Novartis Found Symp 249: 203-217.

Pelttari K, Winter A, Steck E, Goetzke K, Hennig T, Ochs BG, Aigner T, Richter W (2006) Premature induction of hypertrophy during in vitro chondrogenesis of human mesenchymal stem cells correlates with calcification and vascular invasion after ectopic transplantation in SCID mice. Arthritis Rheum 54: 3254-3266.

Radice M, Brun P, Cortivo R, Scapinelli R, Battaliard C, Abatangelo G (2000) Hyaluronan-based biopolymers as delivery vehicles for bone-marrow-derived mesenchymal progenitors. J Biomed Mater Res 50: 101-109.

Revell CM, Athanasiou KA (2009) Success rates and immunologic responses of autogenic, allogenic, and xenogenic treatments to repair articular cartilage defects. Tissue Eng Part B Rev 15: 1-15.

Schagemann JC, Paul S, Casper ME, Rohwedel J, Kramer J, Kaps C, Mittelstaedt H, Fehr M, Reinholz GG 
(2013) Chondrogenic differentiation of bone marrowderived mesenchymal stromal cells via biomimetic and bioactive poly- $\varepsilon$-caprolactone scaffolds. J Biomed Mater Res A 101A: 1620-1628.

Seo JP, Tanabe T, Tsuzuki N, Haneda S, Yamada K, Furuoka H, Tabata Y, Sasaki N (2013) Effects of bilayer gelatin/beta-tricalcium phosphate sponges loaded with mesenchymal stem cells, chondrocytes, bone morphogenetic protein-2, and platelet rich plasma on osteochondral defects of the talus in horses. Res Vet Sci 95: 1210-1216.

Sharma B, Williams CG, Khan M, Manson P, Elisseeff JH (2007) In vivo chondrogenesis of mesenchymal stem cells in a photopolymerized hydrogel. Plast Reconstr Surg 119: $112-120$.

Solchaga LA, Temenoff JS, Gao J, Mikos AG, Caplan AI, Goldberg VM (2005) Repair of osteochondral defects with hyaluronan- and polyester-based scaffolds. Osteoarthritis Cartilage 13: 297-309.

Stark Y, Suck K, Kasper C, Wieland M, van Griensven M, Scheper T (2006) Application of collagen matrices for cartilage tissue engineering. Exp Toxicol Pathol 57: 305311.

Steadman JR, Rodkey WG, Briggs KK (2002) Microfracture to treat full-thickness chondral defects: surgical technique, rehabilitation, and outcomes. J Knee Surg 15: 170-176.

Stoop R (2008) Smart biomaterials for tissue engineering of cartilage. Injury 39 Suppl 1: S77-87.

Tanaka T, Komaki H, Chazono M, Fujii K (2005) Use of a biphasic graft constructed with chondrocytes overlying a $\beta$-tricalcium phosphate block in the treatment of rabbit osteochondral defects. Tissue Eng 11: 331-339.

Tigli RS, Cannizaro C, Gumusderelioglu M, Kaplan DL (2011) Chondrogenesis in perfusion bioreactors using porous silk scaffolds and hESC-derived MSCs. J Biomed Mater Res A 96: 21-28.

van Beuningen HM, Glansbeek HL, van der Kraan PM, van den Berg WB (1998) Differential effects of local application of BMP-2 or TGF-beta 1 on both articular cartilage composition and osteophyte formation. Osteoarthritis Cartilage 6: 306-317.

Williamson AK, Chen AC, Sah RL (2001) Compressive properties and function-composition relationships of developing bovine articular cartilage. J Orthop Res 19: 1113-1121.

Wilson W, Huyghe JM, van Donkelaar CC (2007) Depth-dependent compressive equilibrium properties of articular cartilage explained by its composition. Biomech Model Mechanobiol 6: 43-53.

Xue JX, Gong YY, Zhou GD, Liu W, Cao Y, Zhang WJ (2012) Chondrogenic differentiation of bone marrowderived mesenchymal stem cells induced by acellular cartilage sheets. Biomaterials 33: 5832-5840.

Zhang L, Yuan T, Guo L, Zhang X (2012) An in vitro study of collagen hydrogel to induce the chondrogenic differentiation of mesenchymal stem cells. J Biomed Mater Res A. 100A: 2717-2725.

Zheng L, Sun J, Chen X, Wang G, Jiang B, Fan H, Zhang X (2009) In vivo cartilage engineering with collagen hydrogel and allogenous chondrocytes after diffusion chamber implantation in immunocompetent host. Tissue Eng Part A 15: 2145-2153.

\section{Discussion with Reviewers}

Reviewer II: The scaffolds presented seem to have good potential for cartilage repair. Did the authors consider using these biomaterials in an intraoperative setup without need of cell expansion? One possibility could be for example using the scaffold in combination with marrow stimulation techniques, as already performed in the clinics on a regular basis. That would significantly increase the clinical translation significance of the work.

Authors: We completely agree with the reviewer and we have already added this in our discussion in the revised manuscript by stating "Additionally, we are also considering using the TCP-COL-HA biomaterials in an intraoperative setup that circumvents the need for cell expansion so that the scaffold can, for example, be used in combination with marrow stimulation techniques, which are regularly used in clinics and would enhance the translational potential of the scaffold."

Editor's Note: Scientific Editor in charge of the paper: Mauro Alini. 